\title{
Human Walking Analysis, Evaluation and Classification Based on Motion Capture System
}

\author{
Bofeng Zhang ${ }^{1}$, Susu Jiang ${ }^{1}$, Ke Yan ${ }^{1}$ and Daming Wei, ${ }^{1,2}$ \\ ${ }^{1}$ School of Computer Engineering and Science, Shanghai University \\ ${ }_{2}^{2}$ Professor Emeritus, The University of Aizu, Fukushima, \\ 1P. R. of China \\ ${ }^{2}$ Japan
}

\section{Introduction}

Gait analysis is the systematic study of human walking. It is helpful in the medical management of those diseases which affect the locomotion systems. Recently, the gait motion capture systems are becoming widely used by doctors and physical therapists for kinematics analysis and biomechanics and motion capture research, sports medicine and physical therapy, including human gait analysis and injury rehabilitation. This chapter describes some new progress on human walking analysis that our group made in the past few years based on motion capture system.

Generally, ageing causes many changes to neuromuscular system of a human being, for an example, his walking capabilities degenerate by ageing. Because these changes sometimes result in an increase the number of falls during daily walking, especially after the age of 75, it is very important to study the age related changes in the walking gait of elderly subjects. Many researchers studied stability of human walking gait and it was quoted that human walking gait stability decreases with age increasing the risk of falls in elderly people.

Many studies have been reported about the change in the kinematics parameters with age (Arif et al., 2004). This paper only focuses on the progress of walking modeling and walking stability. Especially, in order to simplify the method of data acquisition, this paper suggests process of reduction on dynamic stability features through feature selection. That will help us analyze stability in a more clear way.

\subsection{Background of walking model}

Various methods are used to overcome the difficulties imposed by the extraction of human gait features. Two approaches are being used for human gait analysis: model-based and non-model-based methods.

The non-model-based method is applied in image-based gait analysis (marker-less analysis). Feature correspondence between successive frames is based upon prediction, velocity, shape, texture and colour. Small motion between consecutive frames is the main assumption, whereby feature correspondence is conducted using various geometric constraints.

For the first one, a priori shape model is established to match real data to this predefined model, and thereby extracting the corresponding features once the best match is obtained. Stick models and volumetric models are the most commonly used methods. The model- 
based approach is the most popular method being used for human motion analysis due to its advantages. It can extract detailed and accurate motion data.

Nash (Nash et al., 1998) proposed a parametric gait model consisting of a pair of articulated lines, jointed at the hip to extract moving articulated objects from a temporal sequence of images. Pendulum model was used to extract and describes human gait for recognition automatically (Cunado et al., 2003). The human leg was modelled as two pendulums joined in series. Zhang (Zhang et al., 2004) proposed a model-based approach to gait recognition by employing a 5-link biped locomotion human model. Akita (Akita, 1984) proposed a model consisting of six segments comprising of two arms, two legs, the torso and the head. Lee (Lee, 2003) suggested a 7-ellipse model, to describe a representation of gait appearance for the purpose of person identification and classification. A 2D stick figure model, which composed of 7 segments, was used to represent the human body, and joint angles and angular velocities are calculated to describe the gait motion (Yoo et la., 2002). Guo (Guo et al., 1994) represented the human body structure in the silhouette by a stick figure model which had 10 sticks articulated with six joints. Cheng (Cheng \& Moura, 1998) represented the human body as a stick figure which was considered to be composed of 12 rigid parts. Dockstader (Dockstader et al, 2002) suggested the use of a hierarchical, structural model of the human body which had 15 points. Rohr (Rohr, 1994) proposed a volumetric model for the analysis of human motion, using 14 elliptical cylinders to model the human body. Karaulova (Karaulova et al., 2000) have used the stick figure model to build a novel hierarchical model of human dynamics represented using hidden Markov models.

\subsection{Background of walking stability}

Theoretically, human walking has rigid periodicity so the next step should repeat the first step strictly. That is to say, all steps must be consistent completely and have no any deference at all. In fact, there is no normal walking pattern and the walking pattern varies from person to person. These walking patterns are considered to be stable until and unless there is an evidence of fall of the person. During walking, human tries to generate periodic series of motions. But due to the physiological limitations, these motions do not re-main exactly periodic but contains some variability or randomness in it. He/she does not try to correct this variability or randomness of these motions if it remains within stability limits. This variability present in the walking patterns is due to not only internal perturbation but also due to external perturbations. The amount of variability present in the walking pattern reflects the quality of neuromuscular control of the human being.

There are many researches which are related to human walking stability. Corriveau et al compare the postural stability of elderly stroke patients with those of healthy elderly people using the distance between the centre of pressure (COP) and the centre of mass (COM) in terms of root mean square. Statistical significance of the COP-COM variable was larger in the stroke group than in healthy subjects, in both the anteroposterior (AP) and mediolateral (ML) directions (Corriveau et al., 2004).

Effect of age on the variability or irregularity of the acceleration of COM in ML, vertical and $\mathrm{AP}$ directions is analyzed by Arif et al, using approximate entropy technique for young and elderly subjects of subjects (Arif et al., 2004).

Literature (Hylton et al., 2003) tried to evaluate acceleration patterns at the head and pelvis in young and older subjects when walking on a level and an irregular walking surface. The subjects are two groups, 30 young people aged 22-39 years (mean 29.0, SD 4.3), and 30 older people with a low risk of falling aged 75-85 years (mean 79.0, SD 3.0). 
The maximum Floquet multipliers (FM) are used to measure orbital stability of upper body in difference walking speed (Marin et al., 2006). Orbital stability changed very little with speed. The general purpose of (Kavanagh, 2006) is to examine factors that may influence acceleration features of the upper body during walking.

Balance in quiet upright stance, which was studied by (Stirling \& Zakynthinaki, 2004), does not imply motionless stability, in fact a ML and AP body sway occurs. Almost $95 \%$ of the anterior-posterior sway happens around the ankle and the hip axis.

Sutherland et al investigated the development of mature walking in children of age from one to seven years old (Sutherland et al., 1980). The objective of literature (PH. Chou, 2003) was to investigate the gait maturation of Taiwan children. Elderly subjects exhibits gait pattern characterized by reduced velocity, shorter step length and increased step timing variability (Hylton et al., 2003). It is mentioned that elderly people reduce their walking speed to improve their walking stability in the literature.

While many walking stability indices have been proposed, there is still no commonly accepted way to define, much less quantify, locomotors stability.

\subsection{Background of walking symmetry}

The concept of gait symmetry itself is no acceptable unified definition, more often many people's research assumes that the normal gait is symmetric, which is to simplify data collection and analysis of gait. In fact gait symmetry was only evaluated with a small number of biomechanical studies that used quantitative data of the two lower limbs. In addition, the gait symmetry was not actually carried out enough exploration. It is because there is no participation of a considerable more number of test objects. One hand, the gait parameter of information provided is the effects of the movement not the reasons of the movement. This may also affect explaining the behaviour of the lower limbs. The other hand, the gait symmetry is not clearly defined, and the use only single gait parameters or a simple statistical method to compare which has made study of gait symmetry been more limited.

Maybe it stands to reason that a healthy man or woman has left leg and right leg symmetrically, and normal gait seems symmetric with ones right-side and left-side. What about the walking stability or dynamic balance if there is not so symmetric? Can we get some quantity or relationship of symmetry and balance?

Gait symmetry is multifaceted. Normal walking seems right-left symmetry because normal people walk with their right foot and left foot. The ability of maintaining balance is essential to keep normal walking. Poor balance is an independent risk factor for falling, so anything that improves balance can have a positive effect on safety and function after stroke. Balance is described as the ability to maintain or move within a weight-bearing posture without falling. We have not found any trends study relating to walking stability and symmetry of human gait. And the trends indicated what meanings.

Yang in his doctoral thesis paper (Yang, 2001) proposed the symmetry indicators of step length and stride length, to describe the step in the role of gait symmetry. But phase symmetry and the step length symmetry are not fully reflects the characteristics of gait, especially the joint angle in the role of symmetry.

\section{Modeling assumptions and theoretical framework}

Walking is a complex dynamic activity. A good human model for gait analysis should be simple, but extensive enough to capture the dynamics of most walkers. 
Almost existing models, which have from 2 segments to more than 15 segments, have two pitfalls. Firstly, they paid more attention to the sagittal plane, and overlook the other two planes, transverse plane and frontal plane. Secondly, it is not enough to describe the particularity of the feet. Most of them regarded the foot as a point. Thus, it is difficult to decide the gait cycle, such as initial heel contact, heel rise, and toe off. So a new walking model, so called Fourteen-Linkage (FL) model, is proposed.

\subsection{Fourteen-linkage model}

DEFINITION 1. (Fourteen-Linkage Model) We suggest a walking model, which is a collection of 19 points, 14 segments and 12 joints, used to specify the position and the configuration of a human body, as shown in Fig. 1.

Position describes the location of a body segment or joint in space, measured in meters. In FL model, the 19 points can be decided by the 30 markers we measured from motion capture system. The relationships between the model and markers are also shown in Fig. 1(a).

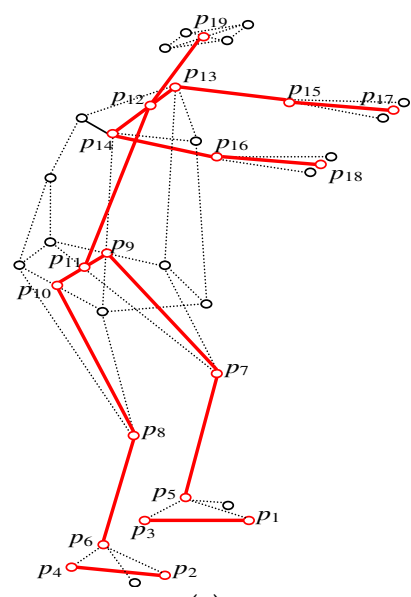

(a)

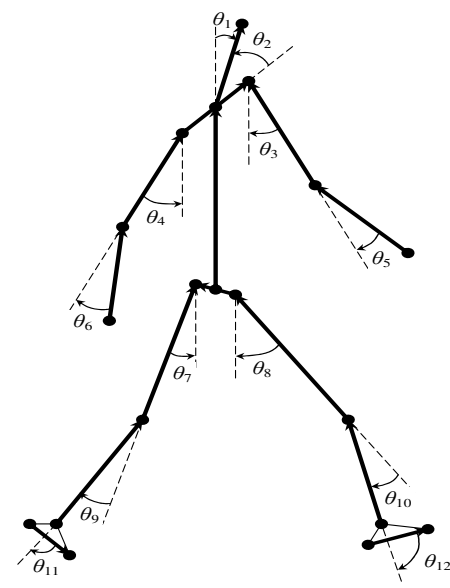

(b)

Fig. 1. Fourteen-linkage (FL) Model

Body segments are considered to be rigid bodies for the purposes of describing the motion of the body. 14 segments are composed of these points, such as head $\left(S_{1}\right)$, shoulder $\left(S_{2}\right)$, left and right upper-arms $\left(S_{3}, S_{4}\right)$, left and right forearms $\left(S_{5}, S_{6}\right)$, trunk $\left(S_{7}\right)$, pelvis $\left(S_{8}\right)$, left and right thighs $\left(S_{9}, S_{10}\right)$, left and right shanks $\left(S_{11}, S_{12}\right)$, and left and right feet $\left(S_{13}, S_{14}\right)$.

12 joints between adjacent segments are composed of these segments, such as head-trunk $\left(\theta_{1}\right)$, head-shoulder $\left(\theta_{2}\right)$, shoulder $\left(\theta_{3}, \theta_{4}\right)$, elbows $\left(\theta_{5}, \theta_{6}\right)$, hips $\left(\theta_{7}, \theta_{8}\right)$, knees $\left(\theta_{9}, \theta_{10}\right)$, ankles $\left(\theta_{11}, \theta_{12}\right)$, see Fig. $1(\mathrm{~b})$.

\subsection{Definitions of walking stability}

In human movement, kinematics is the study of the positions, angles, velocities, and accelerations of body segments and joints during motion.

FL model M consists of displacement $P$, segment angle $\Phi$, joint angle $\Theta$, and their velocity $V$ and acceleration $\mathrm{A}$, angular velocity $\Omega$ and angular acceleration $\Lambda$ at time $\mathrm{t}$, represented as 9-tuple as Equation (1). 


$$
\begin{gathered}
M=[P(t), \Phi(t), \Theta(t), \\
V(t), \Omega_{\Phi}(t), \Omega_{\Theta}(t), \\
\left.A(t), \Lambda_{\Phi}(t), \Lambda_{\Theta}(t)\right]
\end{gathered}
$$

where

$$
\begin{aligned}
& P(t)=\left[p_{1}, p_{2}, p_{3}, \cdots, p_{19}\right]^{T} \\
& \Phi(t)=\left[\varphi_{1}, \varphi_{2}, \varphi_{3}, \cdots, \varphi_{14}\right]^{T} \\
& \Theta(t)=\left[\theta_{1}, \theta_{2}, \theta_{3}, \cdots, \theta_{12}\right]^{T} \\
& V(t)=\left[v_{1}, v_{2}, v_{3}, \cdots, v_{19}\right]^{T} \\
& \Omega_{\Phi}(t)=\left[\omega_{\varphi 1}, \omega_{\varphi 2}, \omega_{\varphi 3}, \cdots, \omega_{\varphi 14}\right]^{T} \\
& \Omega_{\Theta}(t)=\left[\omega_{\theta 1}, \omega_{\theta 2}, \omega_{\theta 3}, \cdots, \omega_{\theta 12}\right]^{T} \\
& A(t)=\left[a_{1}, a_{2}, a_{3}, \cdots, a_{19}\right]^{T} \\
& \Lambda_{\Phi}(t)=\left[a_{\varphi 1}, a_{\varphi 2}, a_{\varphi 3}, \cdots, a_{\varphi 14}\right]^{T} \\
& \Lambda_{\Theta}(t)=\left[a_{\theta 1}, a_{\theta 2}, a_{\theta 3}, \cdots, a_{\theta 12}\right]^{T}
\end{aligned}
$$

Segment angle, as shown in Equation (2), is the angle of the projections of segment with the coordinate axes. It consists of the angles between projections in transverse plane, frontal plane and sagittal plane with axis X, Y and Z respectively, see Fig. 2.

$$
\varphi=\left(\varphi_{x}, \varphi_{y}, \varphi_{z}\right)
$$

Note that it is an absolute measure, meaning that it changes according to the orientation of the body.

Joint angle is the angle between the two segments on either side of the joint. It is defined as Equation (3).

$$
\theta=\left(\theta_{x o y}, \theta_{y o z}, \theta_{z o x}, \theta_{s}\right)
$$

where $\theta_{s}$ is the joint angle in space, and $\theta_{x o y}, \theta_{y o z}, \theta_{z o x}$ are the projections of joint angle in transverse plane, frontal plane and sagittal plane respectively. Since joint angle $\theta_{s}$ is relative to the segment angles, it doesn't change with the body orientation.

Velocity may be linear (change in displacement) or angular (change in angle). Normally, velocity is derived from displacement or angle data by the process of differentiation. Acceleration is change in velocity. Again, it may be linear (change in linear velocity) or angular (change in angular velocity). Acceleration, too, is usually calculated from the displacement data by differentiating twice. It can also be measured directly by an accelerometer. 


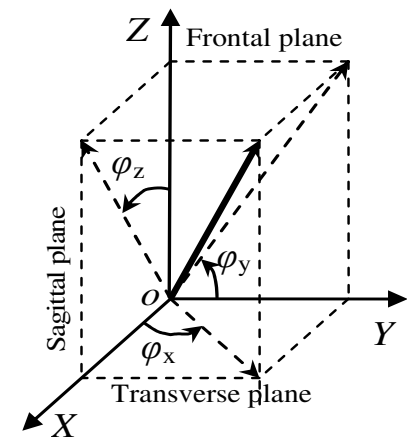

Fig. 2. Segment angle

\subsection{Definitions of walking symmetry}

For walking symmetry, only consider the bilateral of positions, segments and joints on both sides of human body. In FL-Model, there are 8 points, 5 segments and 5 joint angles unilateral met this condition. These attributes are all three-dimensional data. They are motion data and its velocity and acceleration of \{Knee, Ankle, Heel, Toe, Shoulder, Elbow, Wrist, Hip\}, Segment Angle and its velocity and acceleration of \{Thigh, Shank, Foot, Upper-arm, Forearm\}, Joint Angle and its velocity and acceleration of \{Hip, Knee, Ankle, Shoulder, Elbow\}. These attributes can also be expressed with $\mathrm{p}_{1 \sim 10}, \mathrm{p}_{13 \sim 18}, \varphi_{3 \sim 6}, \varphi_{9 \sim 14}, \theta_{3 \sim 12}$, as shown in Fig. 1.

\section{Walking data preprocessing}

Data preprocessing is an important issue for data analysis, as real-world data tend to be incomplete, noisy, and inconsistent. Data preprocessing includes data cleaning, data integration, data transformation, and data reduction. Although numerous methods of data preprocessing have been developed, data preprocessing remains an active area of research, due to the huge amount inconsistent or dirty data and the complexity of the problem. Before we talk about the methods of walking data preprocessing, let us have a look at walking data measuring.

\subsection{Walking data measuring}

There are many measurements of human gait, such as basic data (spatial and temporal data), kinematics (displacement, velocity and acceleration data), kinetics (force and moment data), electromyography (electrical activity of lower limb muscles), and image and graphics (individual silhouette images, monocular, image sequence, video). We adopt the kinematical approach in modeling the human movements.

Two kinds of data, motion data and acceleration data are measured using two different systems. The Motion data are gotten from motion capture system (Vicon MX System by OMG Plc), while acceleration data are obtained by a 3-axis accelerometer.

The providers walk along $5 \mathrm{~m}$ straight line in level plane 3 times at them natural normal walking speeds. 30 markers are attached to the body, as shown in Fig. 3. Fig. 4 shows a snapshot of data acquisition using a motion capture system in the University of Aizu, Fukushima, Japan. The highlights show markers on human body. 


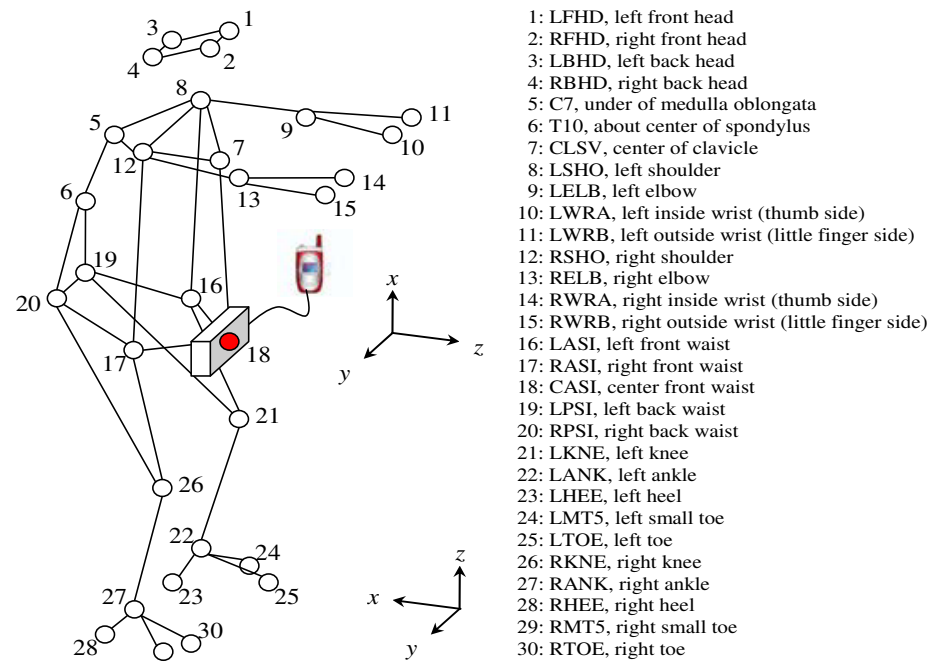

Fig. 3. Markers attached to the body

The motion capture system can detect the three dimensions displacement data at tree directions: anterior-posterior, left-right, and superior-inferior. The sampling rate of motion data is $120 \mathrm{~Hz}$. At the same time, a tri-axial accelerometer unit is mounted with CASI, the same point as marker 18, see Fig. 3. The accelerometer is connected with a mobile phone to save the acceleration data. After that the data can be transferred to computer. Acceleration data are also collected in three dimensions as same as motion data, including the gravity acceleration. But the directions are not same. The sampling rate of acceleration data is $90 \mathrm{~Hz}$. To calibrate the accelerometer, before each testing session, it was placed with each of the orthogonal axes vertically, to estimate the $\pm 1 \mathrm{~g}$ values.

By the way, a movie is taken by a video camera while he/she is walking. 44 normal persons from 20 to 69 year old are measured. These subjects are classified into 5 groups $(20+, 30+$, $40+, 50+$, and $60+$ ) by the age.

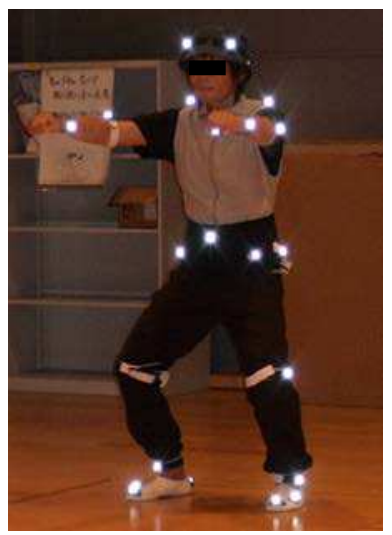

Fig. 4. A snapshot of data acquisition using a motion capture system 


\subsection{Data cleaning}

Data cleaning attempts to fill in missing values, smooth out noise, and correct inconsistencies in the data.

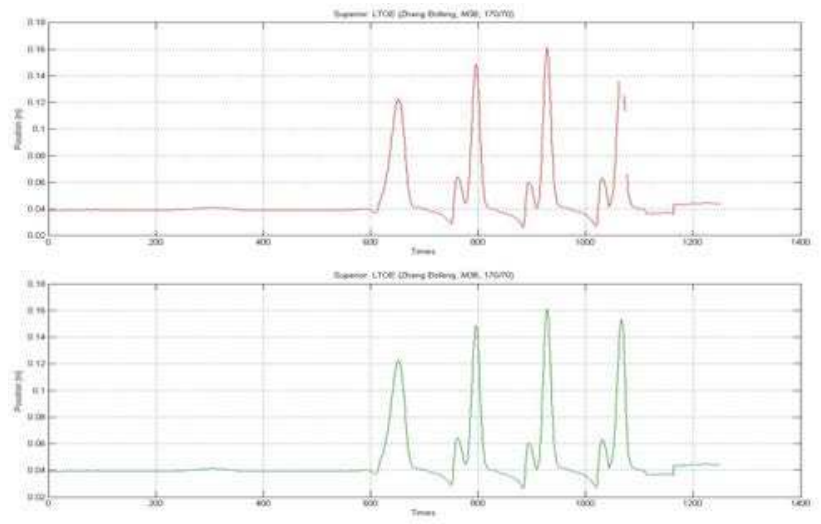

Fig. 5. Interpolation of missing data in left toe

\subsubsection{Missing data}

In the walking data measured by the motion capture systems, there are some missing data because the system can not detect the markers at a moment. Many interpolation methods could be used, such as nearest neighbor interpolation, linear interpolation, cubic spline interpolation, piecewise cubic Hermite interpolation and $\mathrm{N}$-th degree polynomial interpolation. We choose the spline method to interpolate the missing data because the cubic spline interpolation is a piecewise continuous curve, passing through each of the values in the source data. An example is shown in Fig. 5.

\subsubsection{Noisy data}

In acceleration data, there are many noisy data, as shown in Fig. 6. We should try to identify and cut these noisy data from the source data.

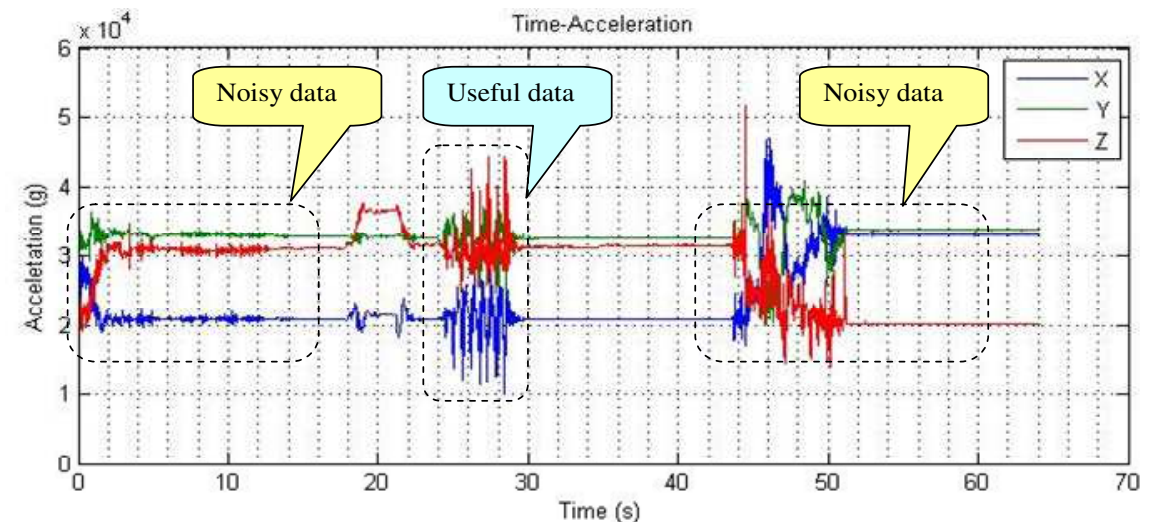

Fig. 6. The noisy data in acceleration data 
And there are some noisy data in motion data caused by vibration. Since the walking signal resides in the low frequency range, it is easily affected by interference from other signal and noise sources. Butterworth low-pass filter is used to reduce noise by passing signal which frequency below twice walking cadence. Some examples are shown in Fig. 7 to Fig. 8.
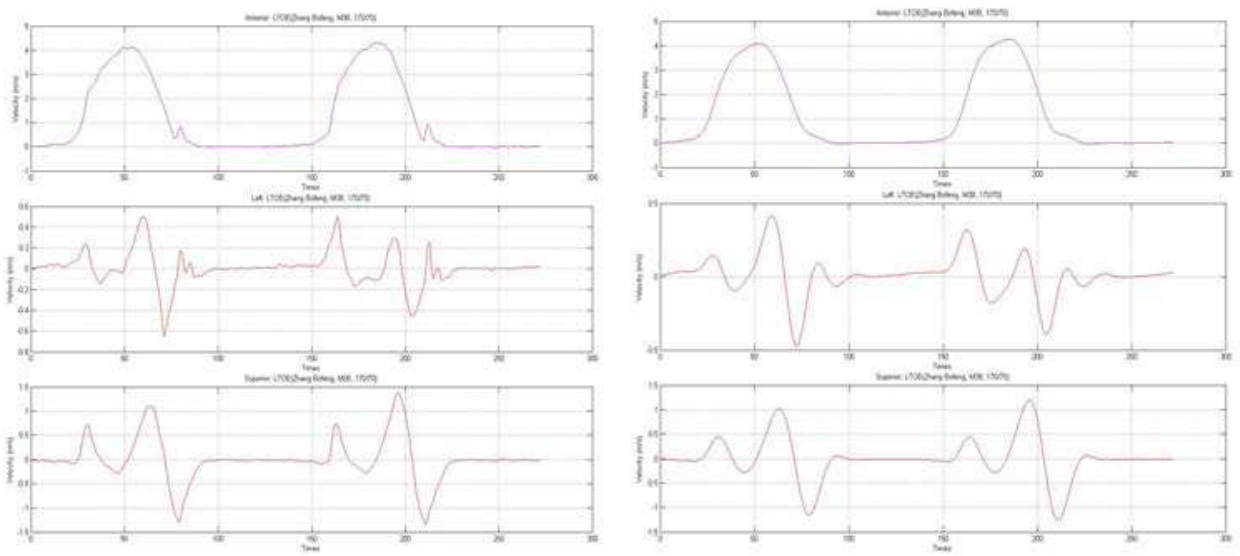

Fig. 7. Velocity data of left toe (No filtering \& Filtering)
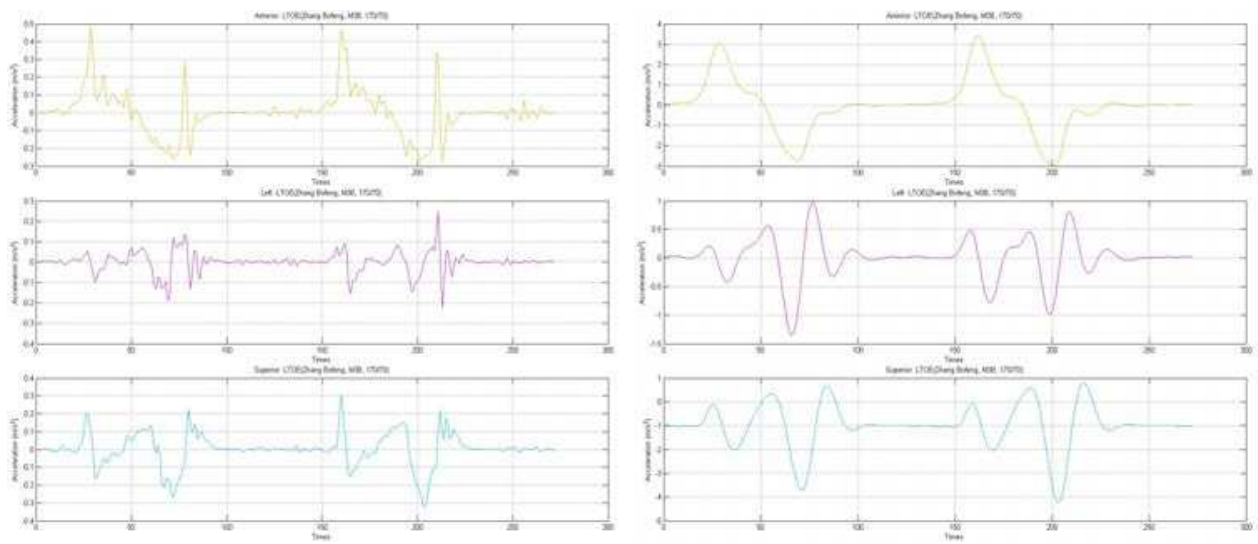

Fig. 8. Acceleration data of left toe (No filtering \& Filtering )

\subsubsection{Inconsistent data}

Because of the faults of the measure systems, one marker can be identified as two or more. In the source file, there is more than one column to store them. So these inconsistent data must be processed by the mean methods, see Equation (4). 


$$
\left\{\begin{array}{l}
\bar{x}=\frac{1}{N} \sum_{i=1}^{N} x_{i} \\
\bar{y}=\frac{1}{N} \sum_{i=1}^{N} y_{i} \\
\bar{z}=\frac{1}{N} \sum_{i=1}^{N} z_{i}
\end{array}\right.
$$

\subsection{Data transformation}

Data transformation converts the data into appropriate forms for analysis further. The coordinate of acceleration data is not parallel the space coordinate because the accelerometer is set up obliquely, so we need to normalize the coordinate.

\subsubsection{Calibration}

The aim of calibration is to transform raw data to acceleration of gravity $(\mathrm{g})$. The standard of vibration amplitude is maintained in terms of electrical output of reference accelerometer corresponding to a known value of displacement. Acceleration singles are sampled at $90 \mathrm{~Hz}$ using purpose-written software and saved on computer for subsequent analysis. To calibrate the accelerations before each testing session, they ware placed with each of the orthogonal axes vertically, first pointing up, then down, which enabled the device to be statically calibrated to estimate the $\pm 1 \mathrm{~g}$ values. An example is shown in Fig. 9.
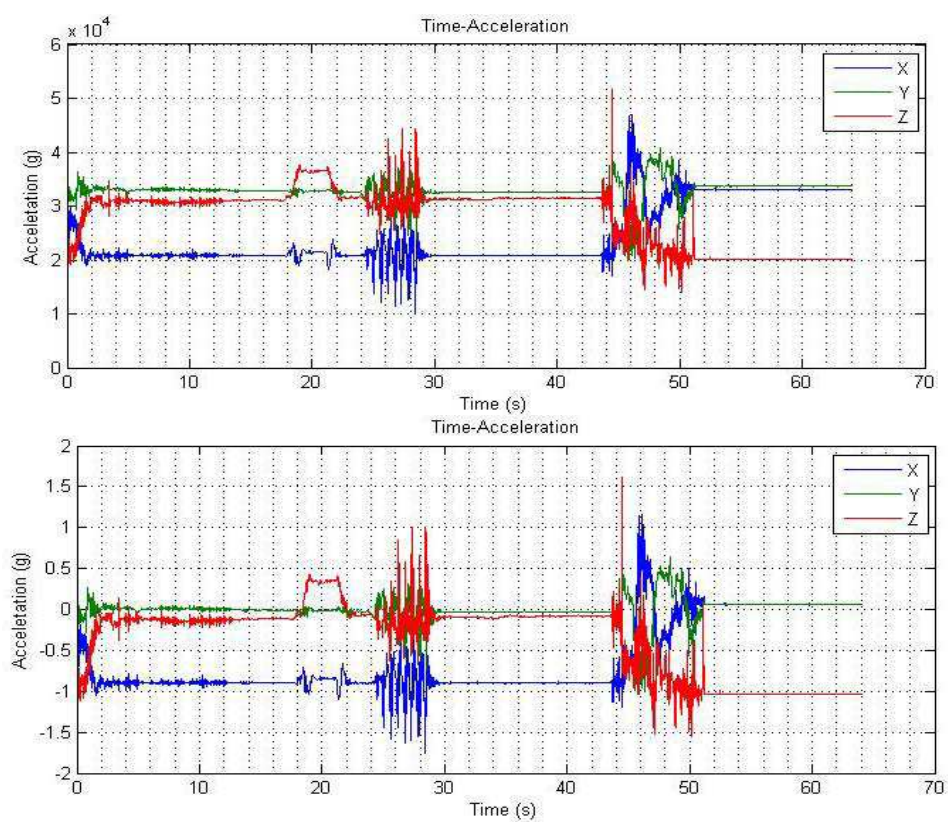

Fig. 9. Calibration of acceleration data 


\subsubsection{Adjusting acceleration data}

Theoretically, the means of $X$ and $Y$ should be ' 0 ', that of $Z$ should be ' -1 '. But actually, it is not true. The coordinate of acceleration data is not parallel with the space coordinate because the accelerometer is set up in gradient, see Fig. 10 so we need to adjust the coordinate.

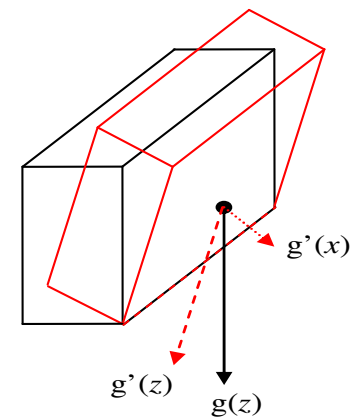

Fig. 10. The accelerometer set up in gradient

The method of adjusting is to rotate the accelerometer in correct position, as shown in Fig. 11. Adjusting rule is that the means of $X$ and $Y$ should be ' 0 '. Firstly, calculate rotation matrix R with Homogeneous Coordinate used Equation (5), and then adjust the acceleration data to vertical position by Equation (6).
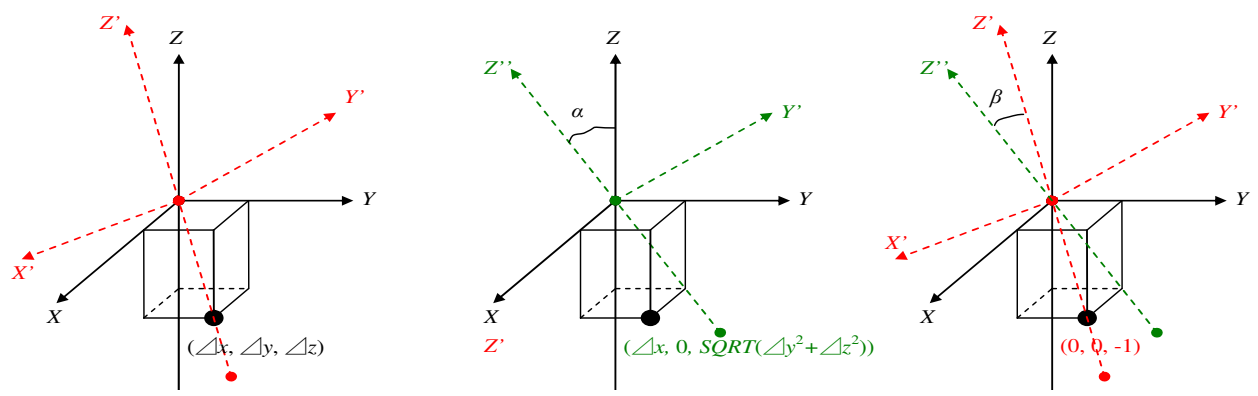

Fig. 11. Title of figure, left justified

$$
\begin{gathered}
R=R_{x} \times R_{y}=\left[\begin{array}{cccc}
1 & 0 & 0 & 0 \\
0 & \cos (\alpha) & \sin (\alpha) & 0 \\
0 & -\sin (\alpha) & \cos (\alpha) & 0 \\
0 & 0 & 0 & 1
\end{array}\right] \times\left[\begin{array}{cccc}
\cos (\beta) & 0 & -\sin (\beta) & 0 \\
0 & 1 & 0 & 0 \\
\sin (\beta) & 0 & \cos (\beta) & 0 \\
0 & 0 & 0 & 1
\end{array}\right] \\
A^{\prime}=A \times R
\end{gathered}
$$

An example of adjusting is shown in Fig. 12. 


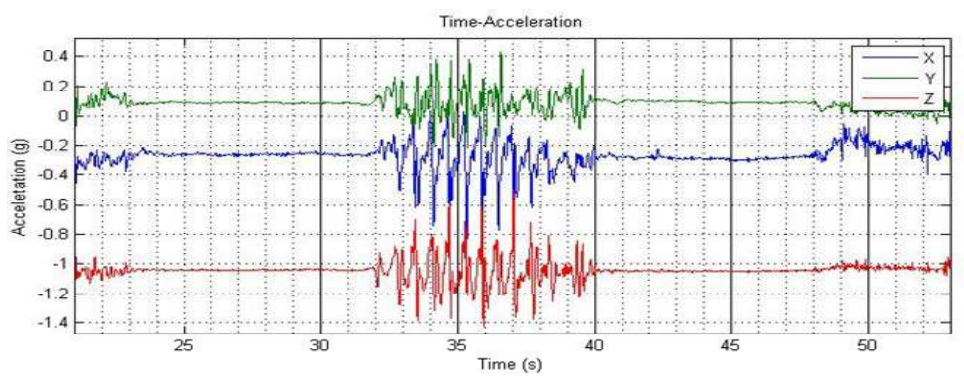

(a) The acceleration data before adjusting

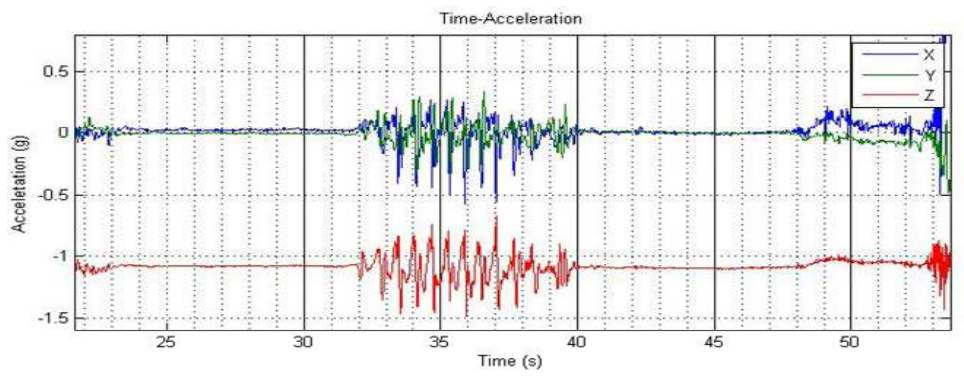

(b) The acceleration data after adjusting

Fig. 12. An example of adjusting result

\subsection{Data integration}

Data integration combines data from multiple sources to form a coherent data store. Metadata, correlation analysis, data conflict detection, and the resolution of semantic heterogeneity contribute toward smooth data integration.

\subsubsection{Converting the coordinates}

The coordinate of acceleration data is different from that of motion data, so we should match the acceleration data and motion data in the same coordinates. We define the coordinates, as shown in Fig. 13, x: anterior, y: left, z: superior.

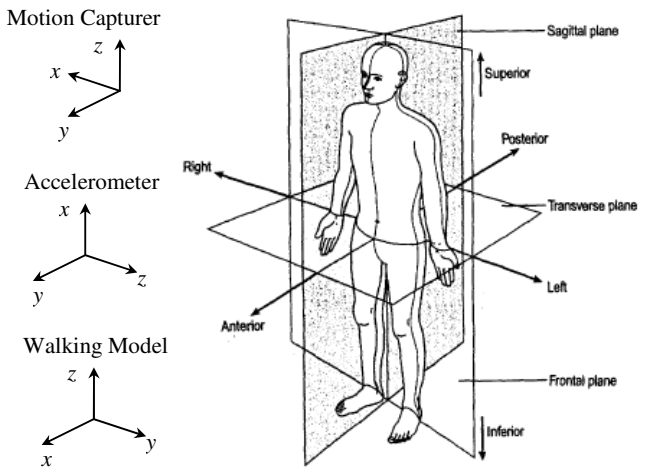

Fig. 13. Converting the coordinates 


\subsubsection{Aligning the acceleration data with the motion data}

The acceleration data and motion data come from different systems, the sampling rates are different, the time of starting measurement are not same, as shown in Fig. 14
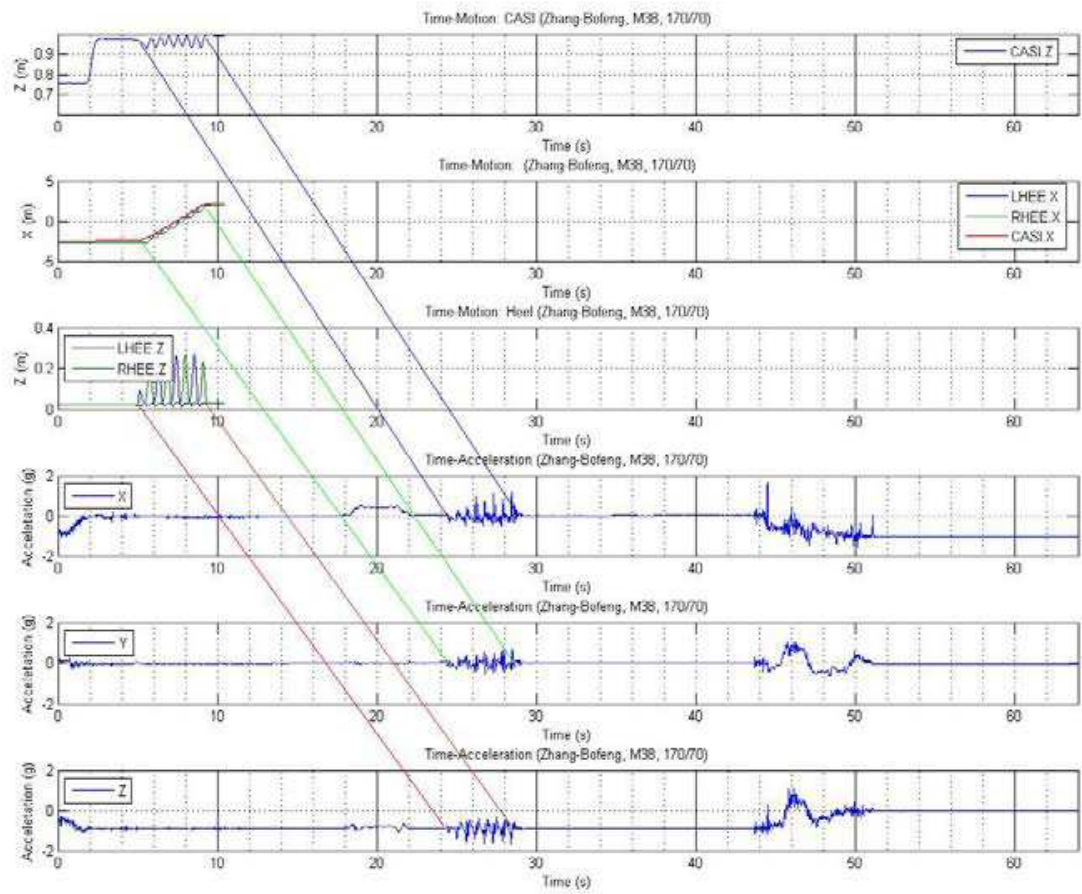

Fig. 14. Title of figure, left justified

so we must find which point of motion data is correlative with which point in acceleration data. The aligning method has two steps.

1. Computing the acceleration with motion data by Equation (7), as shown in Fig. 15.

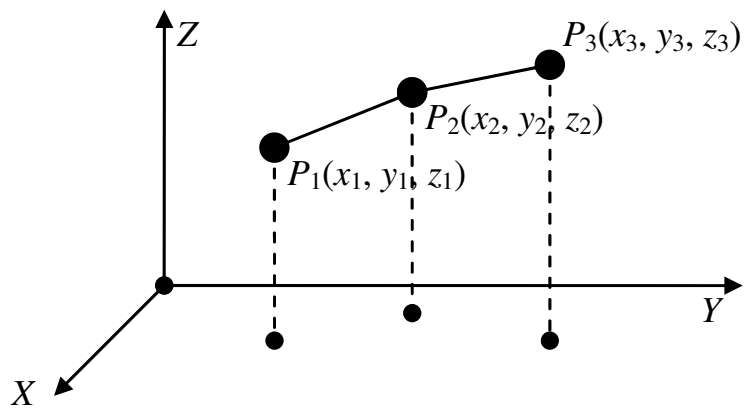

Fig. 15. Computing acceleration with motion data 


$$
\left\{\begin{array}{l}
a_{i}(x)=\frac{x_{i+2}-2 x_{i+1}+x_{i}}{\Delta t^{2}} \\
a_{i}(y)=\frac{y_{i+2}-2 y_{i+1}+y_{i}}{\Delta t^{2}} \quad(i=1, N-2) \\
a_{i}(z)=\frac{z_{i+2}-2 z_{i+1}+z_{i}}{\Delta t^{2}}
\end{array}\right.
$$

2. Comparing the acceleration between the computing data and accelerometer data by minimum of relativity. The result of aligning is shown in Fig. 16.
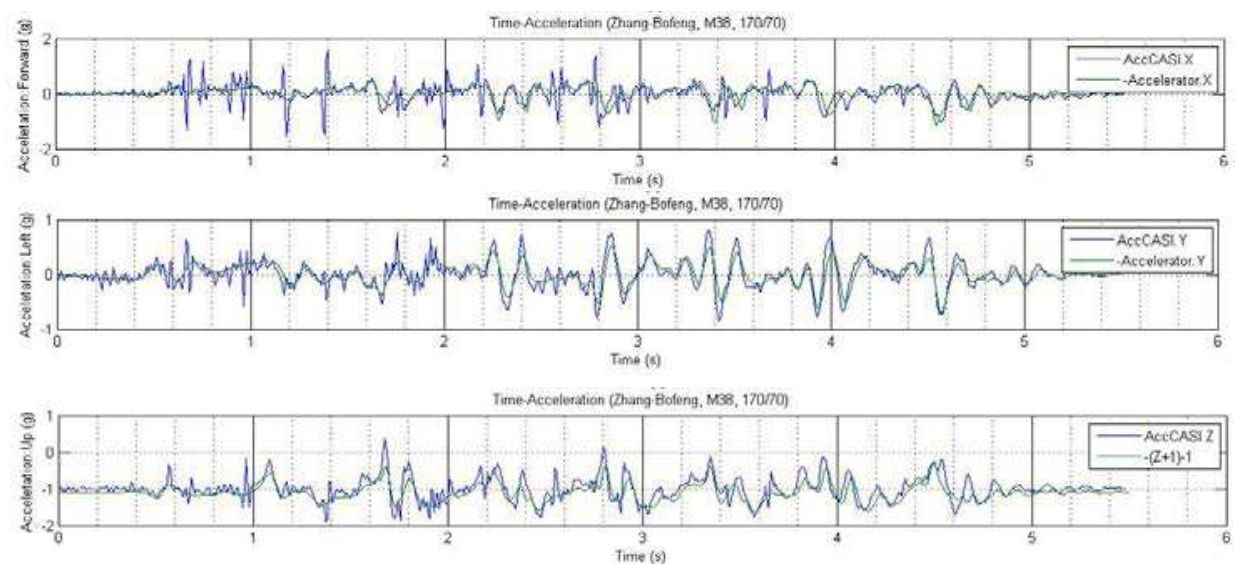

Fig. 16. The result of aligning motion data and acceleration data

\subsection{Data reduction}

Data reduction techniques can be used to obtain a reduced representation of the data while minimizing the loss of information content. To obtain a reduced representation of the data set, such as speed, average span, frequency, and so on, data reduction techniques can be applied, for examples, aggregation operations and conception hierarchy generation. We propose a hierarchical structure shown in Fig. 17.

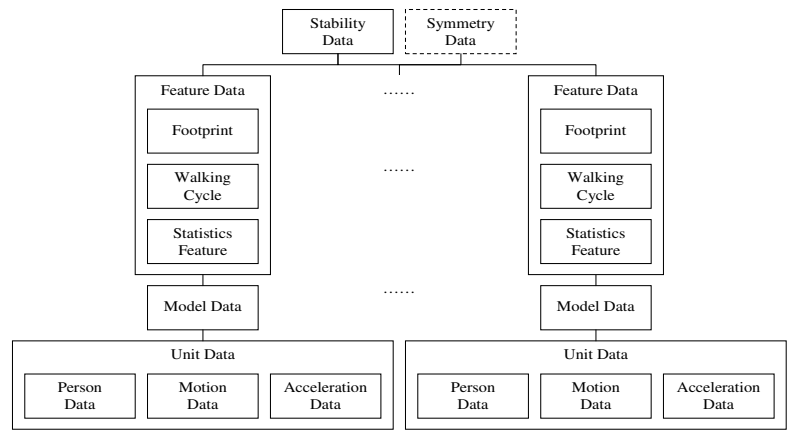

Fig. 17. The hierarchical structure of walking data 


\section{Walking stability analysis}

Although there is now a wealth of literature pertaining to the maintenance of stability when standing, there is a relative paucity of information regarding the biomechanics and physiology of walking stability. Various models are currently in development, but a unified model of walking stability does not exist yet. Unlike standing, the balance of walking is a kind of dynamic balance (Menz, 2000).

Standard deviation is used to define the variability or randomness of the walking pattern. Less the amount of variability means better neuromuscular control and walking stability. We extract a set of hierarchical features from FL model, such as walking cycle features, footprint features.

\subsection{Footprint stability}

Footprint analysis is a typical method of walking research as shown in Fig. 18.

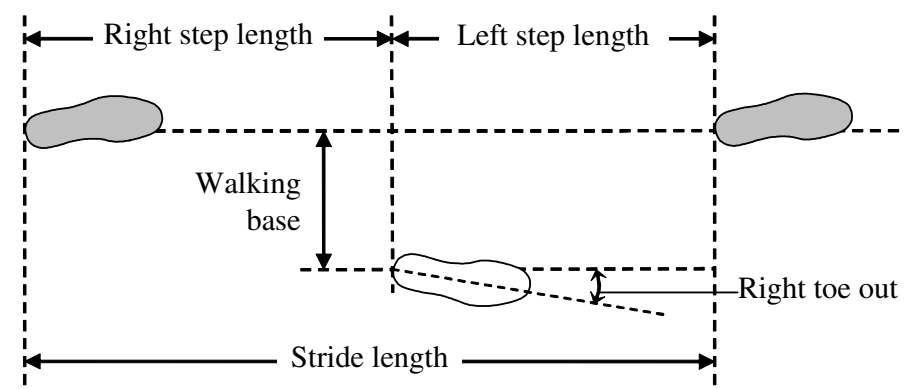

Fig. 18. Features of footprint stability

\subsubsection{Definition of footprint stability}

DEFINITION 2. (Footprint Stability) Footprint stability is described by the variability of the footprint stability features. We extract some of the stability features of footprint $F_{S}(F)$, such as the variability of cycle time $\mathrm{f} 1$, left step length $\mathrm{f} 2$, right step length $\mathrm{f} 3$, speed $\mathrm{f} 4$, walking base f5, left toe out f6, and right toe out f7, as shown in Equation (8).

$$
F_{S}(F)=\sum_{i=1}^{7} \delta\left(f_{i}\right) / \mu\left(f_{i}\right)
$$

where $\delta\left(f_{i}\right)$ is the standard deviation of the feature $f_{i}$, and $\mu\left(f_{i}\right)$ is the mathematical expectation of the feature $f_{i}$.

\subsubsection{Effects of aging on footprint stability}

Now, the variability of the footprint features per decade of age is calculated, as shown in Table 1.

The footprint variability in last row of Table 1 is sum of the above items. It can be seen that the variability is increasing with the age, see Fig. 19. That is to say, the footprint stability is declined with the age, and especially there is a dramatic increasing over 50 years old. But the twenties are exceptional, maybe because the twenties walk more springily than the elders. 


\begin{tabular}{|l|c|c|c|c|c|}
\hline \multicolumn{1}{|c|}{ Age } & $\mathbf{2 0 +}$ & $\mathbf{3 0 +}$ & $\mathbf{4 0 +}$ & $\mathbf{5 0 +}$ & $\mathbf{6 0 +}$ \\
\hline CycleTime & 0.0437 & 0.0293 & 0.0333 & 0.0464 & 0.0716 \\
\hline LStepLength & 0.0825 & 0.0779 & 0.0568 & 0.0766 & 0.0811 \\
\hline RStepLength & 0.0645 & 0.0790 & 0.1099 & 0.0586 & 0.0645 \\
\hline Speed & 0.0833 & 0.0736 & 0.0819 & 0.0709 & 0.0880 \\
\hline WalkingBase & 0.1783 & 0.1721 & 0.1495 & 0.1269 & 0.1343 \\
\hline LToeOut & 0.4047 & 0.1579 & 0.2407 & 0.2383 & 1.3606 \\
\hline RToeOut & 0.3246 & 0.2175 & 0.3473 & 0.5013 & 1.0242 \\
\hline$F_{S}(F)$ & 1.1817 & 0.8073 & 1.0195 & 1.1190 & 2.8243 \\
\hline
\end{tabular}

Table 1. Variability of footprint features

\subsection{Cycle stability}

The gait cycle is defined as the time interval between two successive occurrences of one of the repetitive events of walking. The detection of the human gait period can provide important information to determine the positions of the human body.

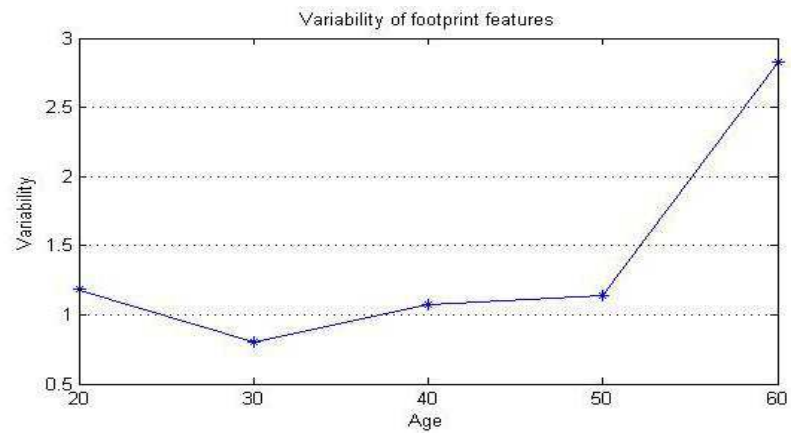

Fig. 19. Footprint stability with age

\subsubsection{Definition of cycle stability}

DEFINITION 3. (Cycle Stability) Cycle stability is described by the variability of the cycle stability features. The cycle stability $F_{S}(C)$ is defined as Equation (9).

$$
F_{S}(C)=\sum_{i=1}^{7} \delta\left(c_{i}\right) / \mu\left(f_{4}\right)
$$

where $\delta\left(c_{i}\right)$ is the standard deviation of the time when event i occurs, $c_{i}=\{\mathrm{LTO}, \mathrm{LFA}, \mathrm{LTV}$, LIC, RTO, RFA, RTV $\}$, and $\mu\left(f_{4}\right)$ is the mathematical expectation of the speed $f_{4}$.

Generally, 7 events are used to identify major events during the walking cycle (Whittle, 2007). For the symmetry of left side and right side, 10 events are employed in this paper, as shown in Fig. 20. 


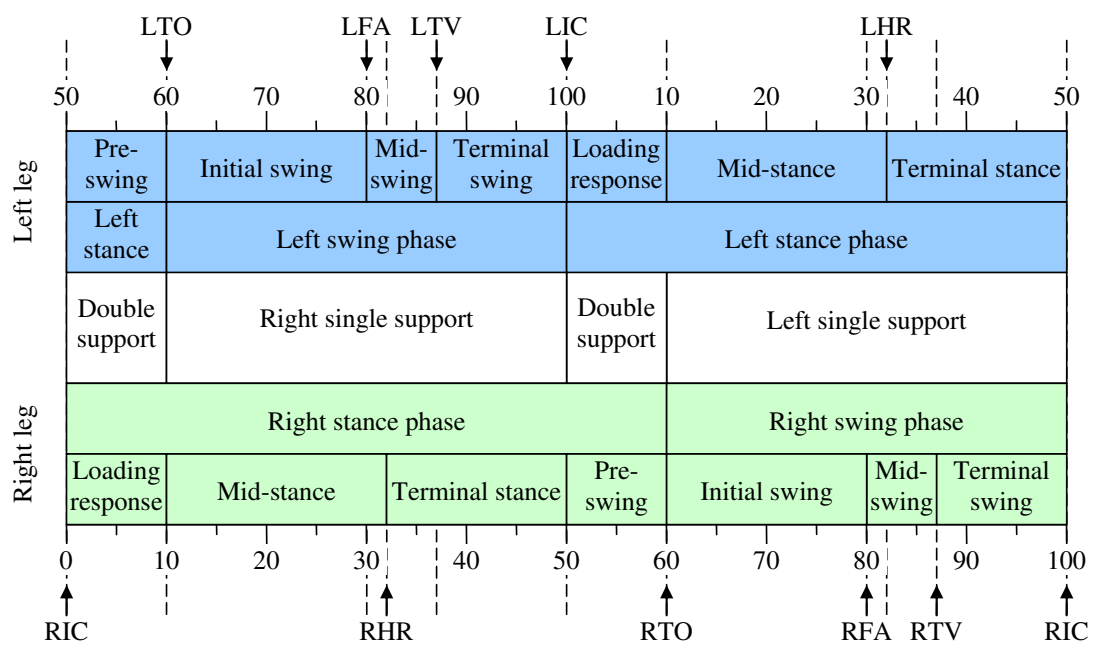

Fig. 20. Walking cycle in normal gait

Such as

RIC = right foot initial contact;

$\mathrm{LTO}=$ left toe off;

LFA = left foot adjacent to right foot;

RHR = right heel rise;

LTV = left tibia vertical;

LIC = left foot initial contact;

$\mathrm{RTO}=$ right toe off;

$\mathrm{RFA}=$ right foot adjacent to left foot;

LHR = left heel rise;

$\mathrm{RTV}=$ right tibia vertical.

\subsubsection{Effects of aging on cycle stability}

Table 2 shows the variability of walking cycle features per decade of age.

\begin{tabular}{|c|c|c|c|c|c|}
\hline Age & $\mathbf{2 0 +}$ & $\mathbf{3 0 +}$ & $\mathbf{4 0 +}$ & $\mathbf{5 0 +}$ & $\mathbf{6 0 +}$ \\
\hline RIC & 0.0000 & 0.0000 & 0.0000 & 0.0000 & 0.0000 \\
\hline LTO & 1.6664 & 1.2287 & 1.2272 & 0.9334 & 1.2268 \\
\hline LFA & 1.2846 & 1.7323 & 1.3302 & 1.0199 & 1.1198 \\
\hline LTV & 1.6354 & 1.8543 & 1.4046 & 1.2759 & 0.9008 \\
\hline LIC & 1.2822 & 1.0320 & 1.0900 & 0.9751 & 0.7036 \\
\hline RTO & 1.3563 & 1.2323 & 1.2866 & 0.7376 & 0.8605 \\
\hline RFA & 0.9731 & 1.3764 & 1.4480 & 1.0737 & 0.8847 \\
\hline RTV & 1.0459 & 1.2067 & 1.3846 & 1.3705 & 1.0909 \\
\hline$F_{S}(C)$ & 9.2440 & 9.6627 & 9.1713 & 7.3861 & 6.7870 \\
\hline
\end{tabular}

Table 2. Variability of walking cycle features 
Fig. 21 shows that the cycle variability is declined with the age almost. This is not same as a common assumption. The reason is that the elder walking more rigidly and inflexibly.

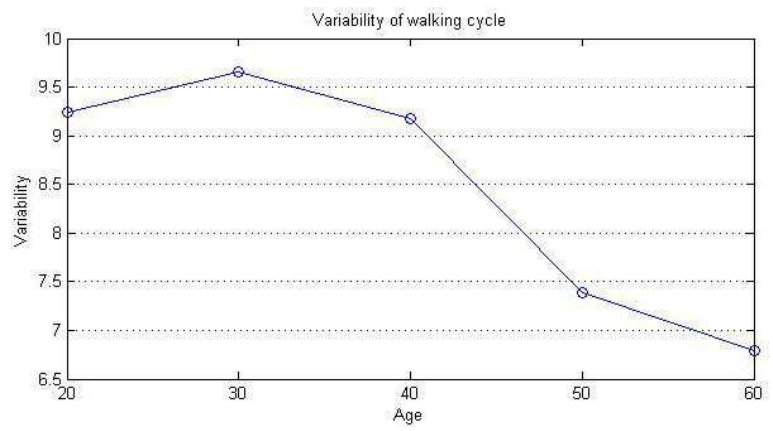

Fig. 21. Cycle stability with age

\subsection{Orbital stability}

Orbital stability defines how purely periodic systems respond to small perturbations discretely after one complete cycle (i.e. one stride) and may be better suited to study strongly periodic movements like walking (Marin, 2006).

\subsubsection{Definition of orbital stability}

DEFINITION 4. (Orbital Stability) Orbital stability is described by the variability of the orbital stability features. The orbital stability $F_{S}(O)$ of lower limbs is composed of three parts, position stability $F_{S}(P)$, segment stability $F_{S}(S)$ and Joint stability $F_{S}(J)$, as shown in Equation (10).

$$
F_{S}(O)=F_{S}(P)+F_{S}(S)+F_{S}(J)
$$

where position stability $F_{S}(P)$ is defined by standard deviation of the maximums and minimums of displacement, velocity and acceleration (knees, heels, and toes), as shown in Equation (11).

$$
\begin{array}{r}
F_{S}(P)=\delta\left[\vee\left(p_{i}(Z)\right)\right]+\delta\left[\vee\left(v_{i}(Z)\right)\right]+\delta\left[\vee\left(A_{i}(Z)\right)\right] \\
+\delta\left[\wedge\left(p_{i}(Z)\right)\right]+\delta\left[\wedge\left(v_{i}(Z)\right)\right]+\delta\left[\wedge\left(A_{i}(Z)\right)\right] \\
(i=1 \sim 4,7,8)
\end{array}
$$

$\checkmark(x)$ and $\wedge(x)$ are the maximum and minimum value of $\mathrm{x}$ respectively.

Segment stability $F_{S}(S)$ is defined by standard deviation of the maximums and minimums of segment angle, angular velocity and angular acceleration (thighs, shanks and feet), as shown in Equation (12).

$$
\begin{array}{r}
F_{S}(S)=\delta\left[\vee\left(\varphi_{i}(Z)\right)\right]+\delta\left[\vee\left(\omega_{\varphi i}(Z)\right)\right]+\delta\left[\vee\left(\alpha_{\varphi i}(Z)\right)\right] \\
+\delta\left[\wedge\left(\varphi_{i}(Z)\right)\right]+\delta\left[\wedge\left(\omega_{\varphi i}(Z)\right)\right]+\delta\left[\wedge\left(\alpha_{\varphi i}(Z)\right)\right] \\
(i=9 \sim 14)
\end{array}
$$

Joint stability $F_{S}(J)$ is defined by standard deviation of the maximums and minimums of displacement, velocity and acceleration (hips, knees and ankles), as shown in Equation (13). 


$$
\begin{array}{r}
F_{S}(J)=\delta\left[\vee\left(\theta_{i}(Z)\right)\right]+\delta\left[\vee\left(\omega_{\theta i}(Z)\right)\right]+\delta\left[\vee\left(\alpha_{\theta i}(Z)\right)\right] \\
+\delta\left[\wedge\left(\theta_{i}(Z)\right)\right]+\delta\left[\wedge\left(\omega_{\theta i}(Z)\right)\right]+\delta\left[\wedge\left(\alpha_{\theta i}(Z)\right)\right] \\
(i=7 \sim 12)
\end{array}
$$

Thus, the orbital stability of lower limbs includes 108 indexes. For examples, Fig.22 and Fig. 23 show the joint angles of hips $\left(\theta_{7}, \theta_{8}\right)$, knees $\left(\theta_{9}, \theta_{10}\right)$ and ankles $\left(\theta_{11}, \theta_{12}\right)$ in sagittal plane during a single walking cycle, their angular velocity $\left(\omega_{\theta 9}, \omega_{\theta 10}, \omega_{\theta 11}, \omega_{\theta 12}\right)$ and angular acceleration $\left(a_{\theta 9}, a_{\theta 10}, a_{\theta 11}, a_{\theta 12}\right)$ respectively.

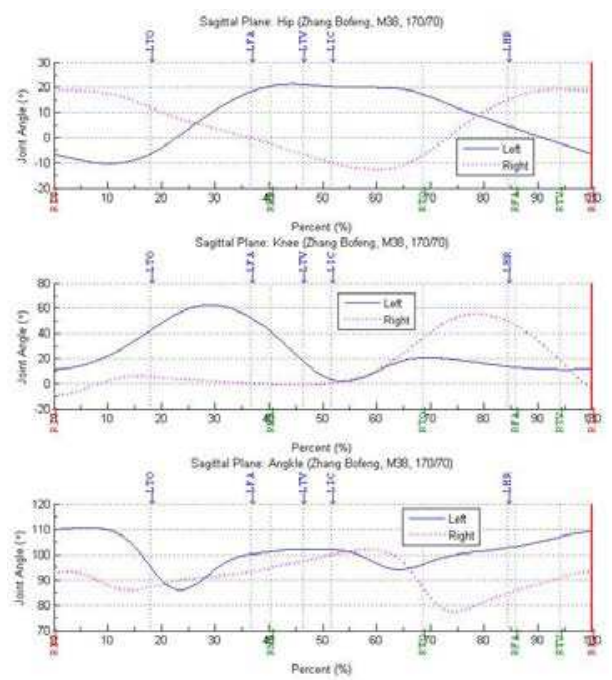

Fig. 22. Joint angles
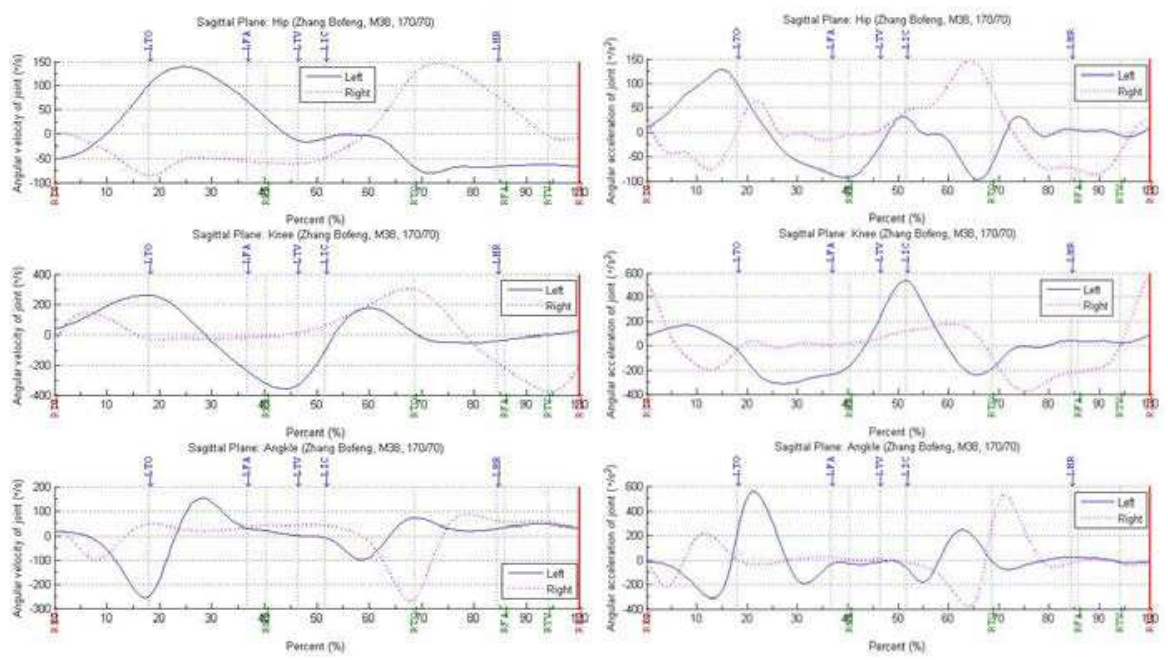

Fig. 23. Angular velocity and acceleration of joint 


\subsubsection{Outlier analysis in orbital stability}

Because of the errors of computing, maybe there are few outliers in the features of orbital stability. For an example, the last 3 cycles of the displacement of right hip (p10) at vertical is shown in Fig. 25. There is an outlier in the last cycle, the left maximum point should be found instead of the right maximum point.

There are a variety of outlier detection approaches from several areas, including statistics, machine learning, and data mining. A kind of proximity-based outlier detection approach, called distance to k-nearest neighbor, is used to find the outliers in orbital stability. This approach is more general and more easily applied than statistical approached, since it is easier to determine a meaningful proximity measure for a data set than to determine its statistics distribution.

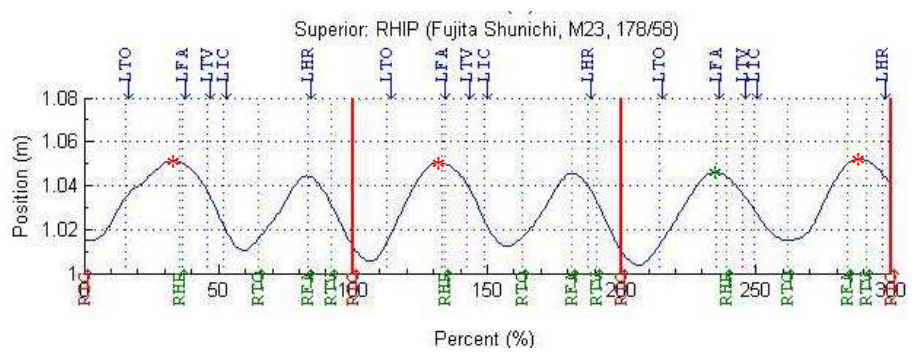

Fig. 24. Last 3 cycles of the displacement of right hip (p10) at vertical

Fig. 25 shows the outliers in the displacement of right hip (p10). The points with a circle are the outlier point. The outlier score of an object is given by the distance to its k-nearest neighbor, using a value of $\mathrm{k}=5$.

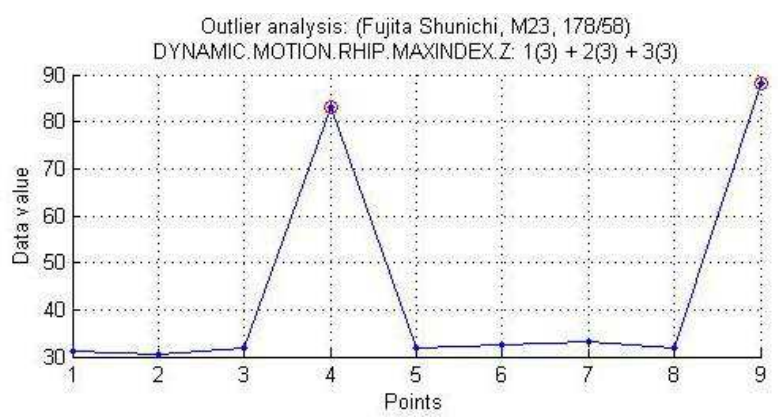

Fig. 25. Outliers in the displacement of right hip

\subsubsection{Effects of aging on orbital stability}

After analysis all subjects between 20 to 70 years old, the item results of orbital stability are shown in Fig. 26. The position stability $F_{S}(P)$, segment stability $F_{S}(S)$, joint stability $F_{S}(J)$ and the whole orbital stability are in shown in Fig. 27. It is observed that, although each of orbital variability does not increase strictly with age, the variability of orbital features increases with age generally. As persons grow old, the orbital stability is becoming weakly. 

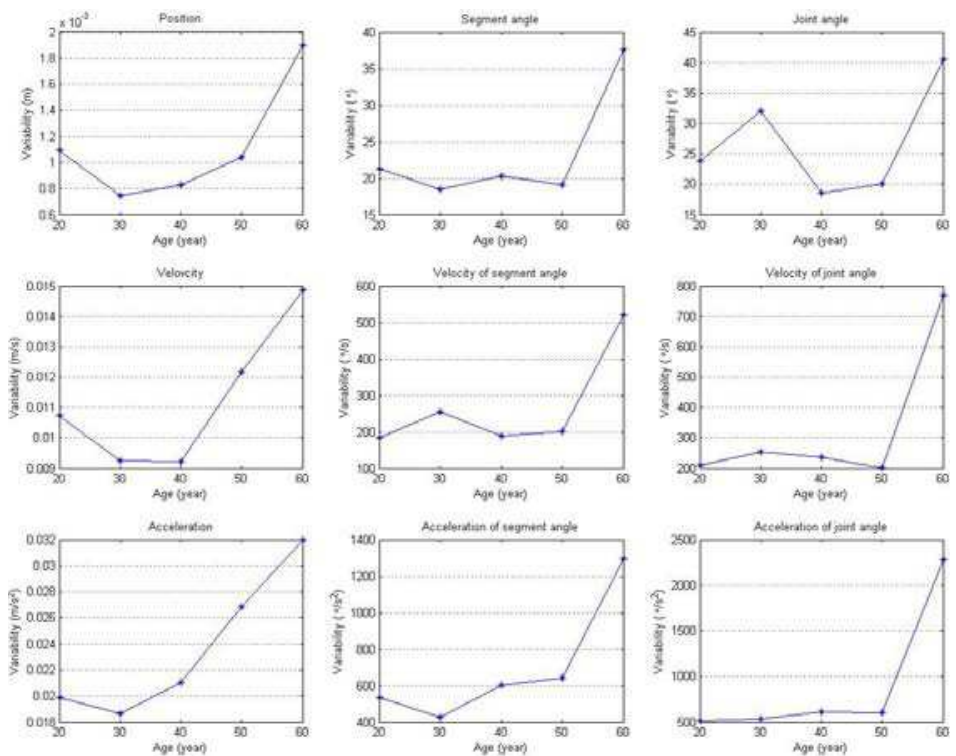

Fig. 26. Each item of orbital stability with age
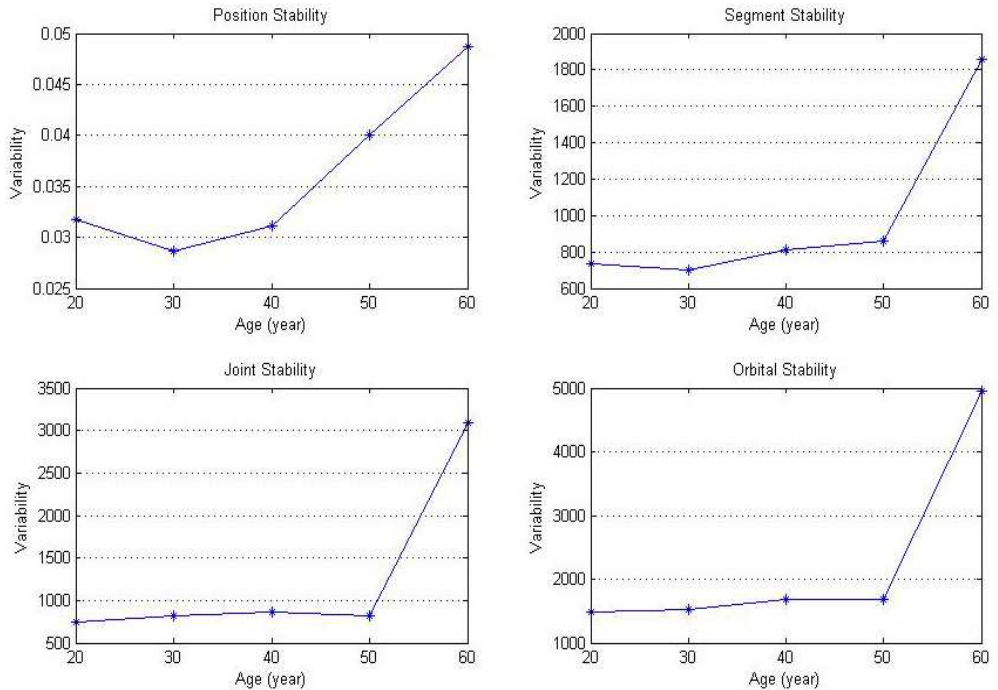

Fig. 27. Position stability, segment stability and joint stability, and orbital stability with age

\subsection{Dynamic stability based on dynamic time warping (DTW)}

Dynamic stability is the main reason for leading to falls for people, especially for elders. This section discusses age influence on dynamic stability based on dynamic time warping. 


\subsubsection{Definition of dynamic stability}

Subject's walking is one kind of periodic movements and the same events will happen during different walking cycles, so the similarity of the data between adjacent cycles to assess subject's walking stability.

This paper used dynamic time warping (DTW) to calculate this similarity, which is a method for flexible pattern-matching scheme. It translates, compress and expands a pair of patterns so similar features within the two patterns are matched (Li, 2003). Fig. 28 and Equation (14) show details.

$$
D\left(S_{i}\right)=\frac{\sum_{j=1}^{n-1} \operatorname{DTW}\left(\theta_{j}, \theta_{j+1}\right)}{n-1}(n>1)
$$

where $D$ is the number of walking cycles, $D\left(S_{i}\right)$ is the average of similarity at feature $S_{i}$.

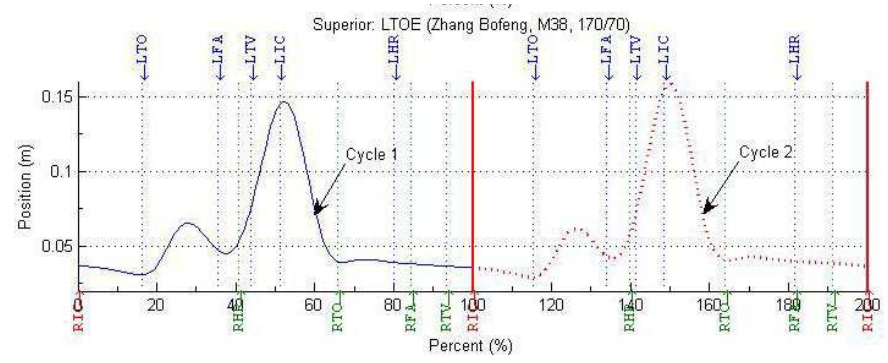

(a) The source data of left toe at superior

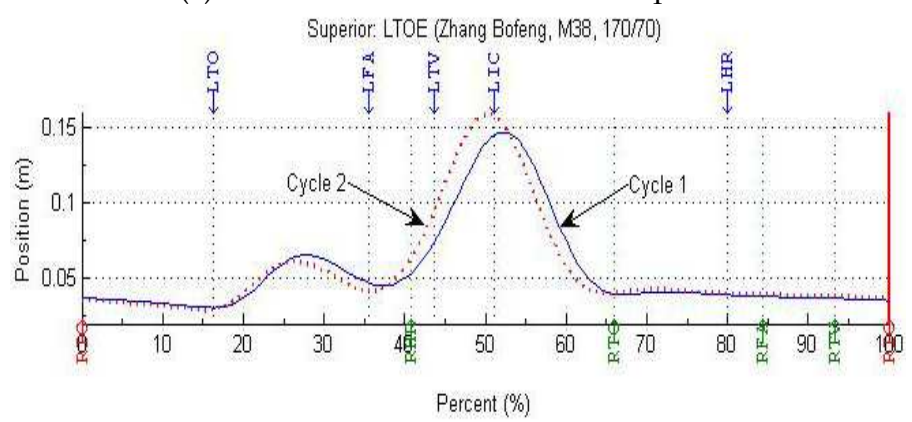

(b) Compare between two adjacent cycles

Fig. 28. Similarity between two adjacent cycles by DTW

\subsubsection{Extracting of dynamic stability features}

According to FL model, it's easy to get corresponding position, velocity and acceleration motion data of 19 points. Therefore, $19 \times 3=57$ features are extracted to describe human dynamic stability.

\subsubsection{Effects of aging on dynamic stability}

Equation (15) calculates the sum of single feature value by age. 


$$
S\left(A_{k}, S_{i}\right)=\frac{1}{m \times l_{k}} \sum_{j=1}^{m} D_{j}\left(S_{i}\right)
$$

where $m$ is the number of subjects in the same age class, $l_{k}$ is the average leg length of subjects in the same age class, $D_{j}\left(S_{i}\right)$ is similarity value of the selected feature $i$ by Equation (14), is a single stability value of the same feature $i$ in the same age class $A_{k}$, and $k$ is the number of age class.

According to Equation (14), similarity value of all selected features in the same age group was calculated by age.

$$
F\left(A_{k}\right)=\sum_{i=1}^{p} S\left(A_{k}, S_{i}\right)
$$

where $p$ is the number of features, in this case, $p$ is equal to 32 , and $k$ is equal to 5 .

Fig. 29 shows trend on the change of dynamic stability.

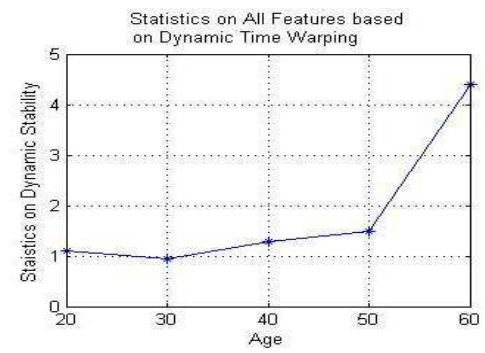

Fig. 29. Statistic on stability of all dynamic features

This figure tells us that if all dynamic features are employed to do statistics on such kind of stability, the dynamic stability is decreasing with ageing except the twenties. It seems that all of those features are desirable.

\subsection{Feature selection on dynamic stability}

The previous section did statistic on all 57 dynamic stability. To reduce the computational complexity, and more importantly, to get more important and contributing features, this paper did feature selection on those 57 dynamic features. On the one hand, it could simplify the method of data acquisition; On the other hand, it is more persuasive by analyzing those selected features.

This section ties to find the best features reflecting the relationship between age and walking stability among those 57 ones, which include $257-1$ different kinds of feature combination. A classic method of feature selection, which is the cooperation of adaptive genetic algorithm and support vector machine, was used to do it.

\subsubsection{Improved crossover operation}

In order to avoid that better solutions with high fitness disappear in a standard genetic algorithm although the algorithm is accommodated again and again. This paper proposed a formulation to adjust crossover probability $\left(p_{c}\right)$ between average fitness and maximum fitness, as shown in Equation (17). 


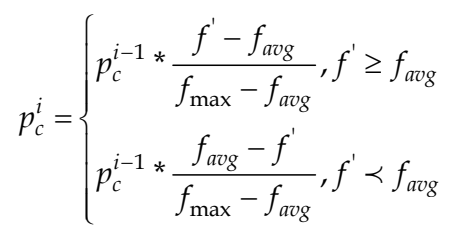

where $f_{\max }$ is the maximum fitness of current population, $f_{\text {avg }}$ is the average fitness of current population, $f^{\prime}$ is the larger fitness between two individuals in crossover operation.

\subsubsection{Improved mutation operation}

Just as crossover operation, there are the same problems with mutation operation.

If probability of mutation $\left(p_{m}\right)$ is undersize, new individual can't be generated easily, inversely, genetic algorithm will be a pure searching process.

To solve this problem, this paper improved it as shown in Equation (18).

$$
p_{m}^{i}=\left\{\begin{array}{l}
p_{m}^{i-1} * \frac{f-f_{\text {avg }}}{f_{\max }-f_{\text {avg }}}, f \geq f_{\text {avg }} \\
p_{m}^{i-1} * \frac{f_{\text {avg }}-f}{f_{\max }-f_{\text {avg }}}, f \prec f_{\text {avg }}
\end{array}\right.
$$

where $f$ is the fitness of individual going to mutate. All other parameters have the same meaning as Equation (17).

\subsubsection{Improved support vector machine (SVM)}

According to information of age classification, SVM was used to separate datasets and assess fitness of specific feature combination during feature selection. This paper improves SVM in two parts: classification balancing and evaluation.

A conventional SVM is to build a decision function $f_{c}(x)$ for each class $C$. Then use Equation (19) as the predicted class label.

$$
d(x)=\arg \max \left(f_{\mathrm{c}}(x)\right)
$$

However, this equation may fail to work in some skewed inseparable distribution. Therefore, it's improved as Equation (20), which suggests a function $p_{c}(f)$ to balance values of $f_{c}(x)$ in Equation (19).

$$
d(x)=\arg \max \left(p_{c}\left(f_{\mathrm{c}}(x)\right)\right)
$$

Another problem is about evaluation. Generally, correctness is calculated by correctNumber/totalNumber, but it does not fit well to skewed distributions. It's improved as Equation (21) described.

$$
F(M, b, \vec{w})=1-(1-b) \times \sum_{i=1}^{n} \frac{e_{i}}{m}-U
$$




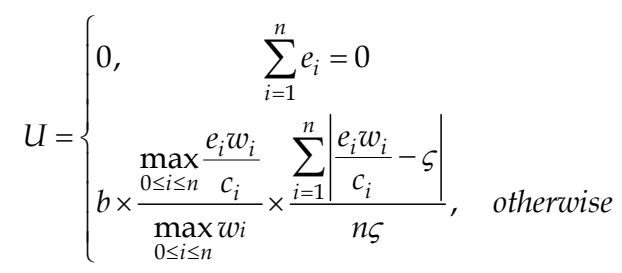

where $M$ is the confusion matrix, $b$ is a coefficient that balances the total correctness and the balance achievement, $\vec{w}$ is the importance weight between classes, $n$ is the number of classes, $e_{i}$ is the sum of non-diagonal entries of $i$-th row of $M, m$ is the sum of all entries of $M$, namely total number of patterns, $\sum_{i=1}^{n} w_{i}=n, \varsigma$ is the average value of $e_{i} w_{i} / c_{i}$.

\subsubsection{Selected stable features}

After 18 generations of GA, 32 walking stability features were selected with classification correctness from $89.4 \%$ to $94.7 \%$.

To compare number of markers between before and after feature selection, those 32 features are marked with 14 red markers, as shown in Fig. 30. It means that there is $(30-14) / 30=53.3 \%$ reduction on markers, which could simplify equipment to a large extent.

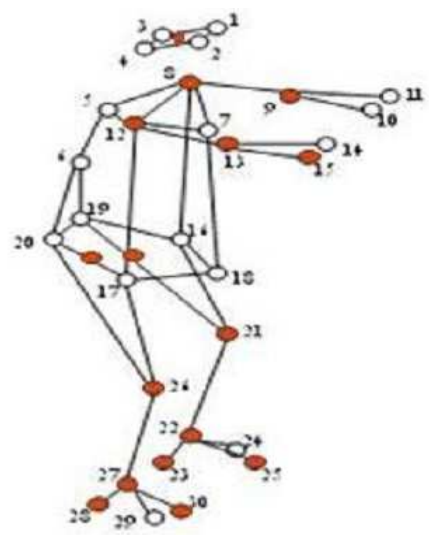

Fig. 30. Red markers corresponding to selected features

\subsubsection{Effects of aging on dynamic stability after feature selection}

Fig. 30 told us that the selected markers are almost symmetrical. To increase the symmetry, other three features are added, such as LHIP in position, RELB in velocity, RSHO and LELB in acceleration. Table 3 shows these fours features with gray background in details.

The difference between this method and previous one is the number of dynamic features. Therefore, the same statistic method will be applied on this one. Another, the similarity calculated by DTW is used to assess the stability of specific dynamic feature. Because DTW doesn't care about the data unite, all features from position, velocity and acceleration will be counted together. 


\begin{tabular}{|c|c|c|c|c|c|}
\hline Age & $20+$ & $30+$ & $40+$ & $50+$ & $60+$ \\
\hline Position & & & & & \\
\hline LHIP & 0.0001 & 0.0000 & 0.0001 & 0.0001 & 0.0003 \\
\hline RHIP & 0.0001 & 0.0001 & 0.0001 & 0.0001 & 0.0003 \\
\hline CENT & 0.0001 & 0.0000 & 0.0001 & 0.0001 & 0.0003 \\
\hline LKNE & 0.0002 & 0.0005 & 0.0001 & 0.0006 & 0.0003 \\
\hline RKNE & 0.0002 & 0.0002 & 0.0001 & 0.0004 & 0.0013 \\
\hline LANK & 0.0012 & 0.0010 & 0.0001 & 0.0001 & 0.0009 \\
\hline RANK & 0.0013 & 0.0006 & 0.0001 & 0.0001 & 0.0005 \\
\hline LHEE & 0.0028 & 0.0027 & 0.0001 & 0.0002 & 0.0013 \\
\hline RHEE & 0.0027 & 0.0018 & 0.0000 & 0.0001 & 0.0011 \\
\hline LTOE & 0.0004 & 0.0001 & 0.0001 & 0.0001 & 0.0002 \\
\hline RTOE & 0.0004 & 0.0002 & 0.0001 & 0.0001 & 0.0002 \\
\hline Velocity & & & & & \\
\hline LELB & 0.0125 & 0.0023 & 0.0122 & 0.0046 & 0.0055 \\
\hline RELB & 0.0111 & 0.0030 & 0.0080 & 0.0039 & 0.0055 \\
\hline CENT & 0.0021 & 0.0009 & 0.0016 & 0.0018 & 0.0024 \\
\hline LKNE & 0.0109 & 0.0077 & 0.0038 & 0.0035 & 0.0066 \\
\hline RKNE & 0.0109 & 0.0076 & 0.0024 & 0.0034 & 0.0054 \\
\hline LANK & 0.0563 & 0.0405 & 0.0088 & 0.0091 & 0.0495 \\
\hline RANK & 0.0678 & 0.0318 & 0.0594 & 0.0062 & 0.1520 \\
\hline LHEE & 0.1522 & 0.1369 & 0.0055 & 0.0200 & 0.0775 \\
\hline RHEE & 0.1447 & 0.0892 & 0.0073 & 0.0847 & 0.0662 \\
\hline LTOE & 0.0336 & 0.0135 & 0.0067 & 0.0079 & 0.0158 \\
\hline RTOE & 0.0400 & 0.0201 & 0.0129 & 0.0569 & 0.0204 \\
\hline Acceleration & & & & & \\
\hline LSHO & 0.0071 & 0.0030 & 0.0041 & 0.0061 & 0.0037 \\
\hline RSHO & 0.0071 & 0.0033 & 0.0047 & 0.0054 & 0.0035 \\
\hline NECK & 0.0062 & 0.0028 & 0.0038 & 0.0051 & 0.0031 \\
\hline LELB & 0.0162 & 0.0046 & 0.0230 & 0.0080 & 0.0069 \\
\hline RELB & 0.0137 & 0.0045 & 0.0094 & 0.0080 & 0.0077 \\
\hline CENT & 0.0059 & 0.0029 & 0.0054 & 0.0349 & 0.0449 \\
\hline LKNE & 0.0293 & 0.0179 & 0.0214 & 0.0119 & 0.0161 \\
\hline RKNE & 0.0299 & 0.0187 & 0.0095 & 0.0136 & 0.0204 \\
\hline LANK & 0.0765 & 0.0505 & 0.0342 & 0.0432 & 0.0562 \\
\hline RANK & 0.0915 & 0.0544 & 0.4998 & 0.0270 & 2.2505 \\
\hline LHEE & 0.1604 & 0.1196 & 0.0812 & 0.0901 & 0.0887 \\
\hline RHEE & 0.1662 & 0.1154 & 0.0477 & 0.2507 & 0.0980 \\
\hline LTOE & 0.1567 & 0.0687 & 0.0274 & 0.2517 & 0.0965 \\
\hline RTOE & 0.2266 & 0.1225 & 0.0816 & 0.2483 & 0.1541 \\
\hline F(A $)$ & $\mathbf{1 . 5 4 4 9}$ & $\mathbf{0 . 9 4 9 7}$ & $\mathbf{1 . 0 0 2 8}$ & $\mathbf{1 . 0 9 8 0}$ & 3.2639 \\
\hline & & & & & \\
\hline
\end{tabular}

Table 3. Selected features in three types 


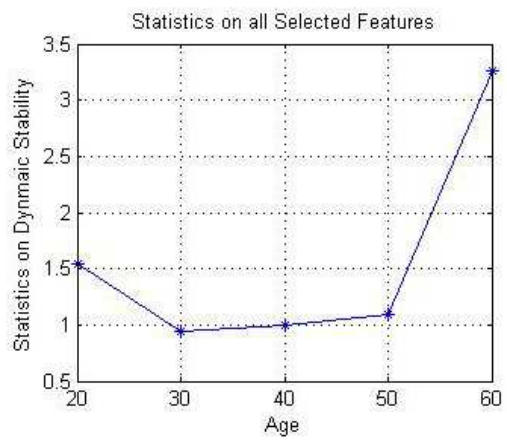

Fig. 31. Statistic on stability of selected dynamic features

As a response to the assumption that elder the person is, less stable his gait is. This method assesses walking stability by searching the best contributing features and doing statistics on them. The result shows that walking stability truly becomes worse as ageing except the group of twenties.

\section{Walking symmetry analysis}

Gait symmetry analysis is a part of normal gait analysis. Our research is based on the Fourteen-Linkage Walking Model of human. The detail of this model can be seen in chapter 2. We all know, gait symmetry reflects the general characteristics of human walk gait, and it is an important indicator to assess the function of the human walk. Especially in the human aging process the recession of brain and central nervous system and physiological function will affect the lower limb gait of left or right side, and lead gait mutation.

\subsection{Footprint symmetry}

Here, we mainly aim to investigate the footprint properties of the left and right foot. See Fig.32, the symmetric properties were step length and toe out angle of two feet.

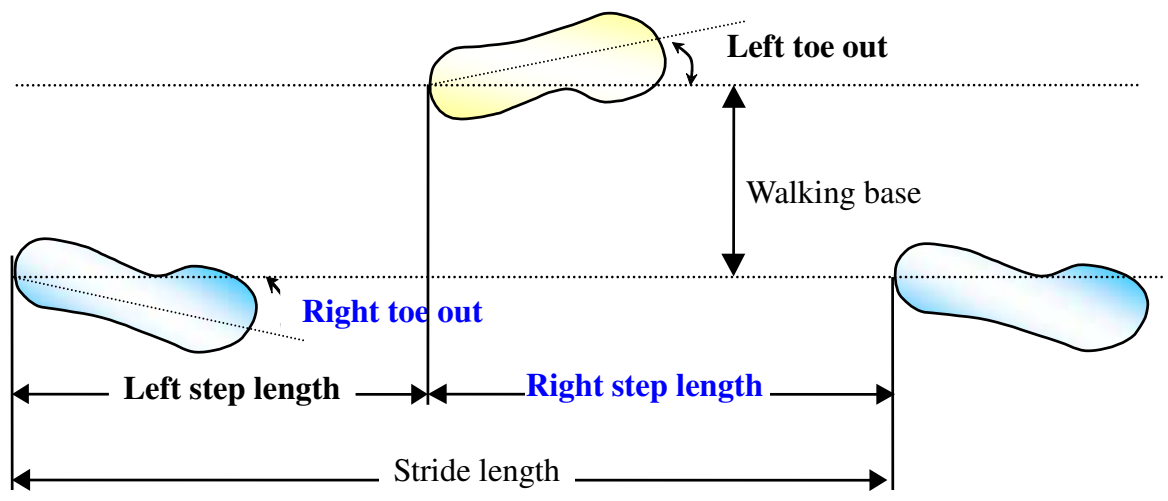

Fig. 32. Features of footprint for symmetry analysis 
Besides step length and toe out angle, we also consider step factor and walk ratio.

Step factor is the value of step length divided by leg length.

Walk ratio is the value of step length divided by step rate and step rate is the number of steps per minute someone walks.

\subsubsection{Definition of footprint symmetry}

DEFINITION 5. (Footprint Symmetry) Footprint symmetry is described by the difference of the bilateral footprint features. We extract some symmetrical features of footprint as $S(F)$, which contains the difference of Step length about two feet, the difference of Toe out angle of right foot and left foot, the difference of bilateral Step factor and the difference of bilateral Walk ratio, as shown in Equation (22).

$$
S(\boldsymbol{F})=\sum_{i=1}^{4}\left|\delta\left(L A_{i}\right) / \mu\left(L A_{i}\right)-\delta\left(R A_{i}\right) / \mu\left(R A_{i}\right)\right|
$$

Here $\delta\left(A_{i}\right)$ is the standard deviation of the feature $A_{i}$, and $\mu\left(A_{i}\right)$ is the mathematical expectation of the feature $A_{i} . A_{i}$ is the element of \{LStepLength, RStepLength; LToeout, RToeout; LStepFactor, RStepFactor; LWalkRatio, RWalkRatio\}.

The $\delta / \mu$ of footprint bilateral features per decade of age is calculated, shown as the upper part in Table 4 . The last row of Table 4 is the $S(F)$ of above items.

\subsubsection{Effects of aging on footprint symmetry}

It can be seen that the variation of footprint feature is mostly less than 0.02 excepted on feature Toeout as shown in Fig. 33.

\begin{tabular}{|l|c|c|c|c|c|}
\hline \multicolumn{1}{|c|}{ Age } & $\mathbf{2 0 +}$ & $\mathbf{3 0 +}$ & $\mathbf{4 0 +}$ & $\mathbf{5 0 +}$ & $\mathbf{6 0 +}$ \\
\hline S(StepLength) & 0.01818 & 0.00865 & 0.00238 & 0.01286 & 0.00210 \\
\hline S(Toeout) & 0.01660 & 0.04051 & 0.04079 & 0.16155 & 0.05628 \\
\hline S(StepFactor) & 0.01803 & 0.00859 & 0.00247 & 0.01157 & 0.00298 \\
\hline S(WalkRatio) & 0.00814 & 0.01854 & 0.00939 & 0.00293 & 0.00638 \\
\hline S(F) & $\mathbf{0 . 0 6 0 9 5}$ & $\mathbf{0 . 0 7 6 3}$ & $\mathbf{0 . 0 5 5 0 4}$ & $\mathbf{0 . 1 8 8 9 2}$ & $\mathbf{0 . 0 6 7 7 4}$ \\
\hline
\end{tabular}

Table 4. Variation of footprint features

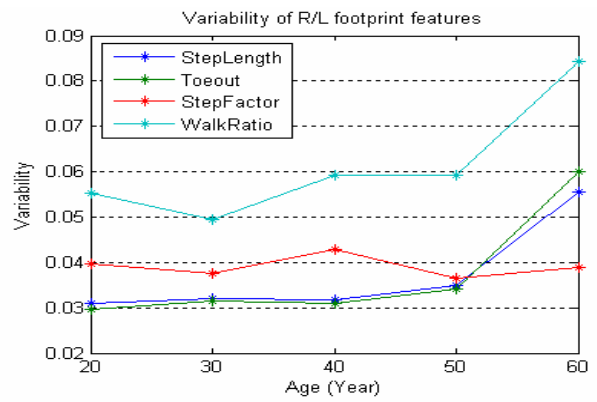

Fig. 33. Variation of footprint features 


\subsection{Cycle symmetry}

This section shows the main characteristics of the symmetry of the gait cycles. As movement of two legs turns symmetrically, the ten tags of walking cycle (see Fig. 20) in fact is a symmetrical composition of 5 keywords in one side, they are TO, FA, TV, IC, HR.

\subsubsection{Definition of cycle symmetry}

DEFINITION 6. (Cycle Symmetry) Cycle symmetry is described by the variability of the cycle symmetric features. The cycle symmetry $S(C)$ is defined as Equation (23).

$$
\mathbf{S}(\mathbf{C})=\sum_{i=1}^{4}\left|\delta\left(L C_{i}\right) / \mu\left(L C_{i}\right)-\delta\left(R C_{i}\right) / \mu\left(R C_{i}\right)\right| / \mu\left(S_{i}\right)
$$

Here $\delta\left(C_{i}\right)$ is the standard deviation of the feature $C_{i}$ and $\mu\left(C_{i}\right)$ is its mathematical expectation, and $\mu\left(S_{i}\right)$ is the mathematical expectation of the feature Speed. $L C_{i}$ or $R C_{i}$ is the element of $\{$ LTO, RTO; LHR, RHR; LFA, RFA; LTV, RTV\}. Since the use of the relative value of sagittal plane, so the IC in here is meaningless.

\subsubsection{Effects of aging on cycle symmetry}

Here described left and right foot gait cycle symmetrical properties change with aging groups. We calculated the $\delta / \mu$ on average speed of every decade of age, as shown in Table 5 .

\begin{tabular}{|l|l|l|l|l|l|}
\hline \multicolumn{1}{|c|}{ Age } & \multicolumn{1}{|c|}{$\mathbf{2 0 +}$} & $\mathbf{3 0 +}$ & \multicolumn{1}{|c|}{$\mathbf{4 0 +}$} & $\mathbf{5 0 +}$ & \multicolumn{1}{c|}{$\mathbf{6 0 +}$} \\
\hline$S(T O)$ & 0.05567 & 0.10183 & 0.04917 & 0.03336 & 0.05064 \\
\hline$S(F A)$ & 0.01323 & 0.01264 & 0.0179 & 0.01706 & 0.01161 \\
\hline$S(H R)$ & 0.03106 & 0.01222 & 0.03407 & 0.01832 & 0.02689 \\
\hline$S(T V)$ & 0.00201 & 0.03435 & 0.01366 & 0.01486 & 0.00648 \\
\hline$S(C)$ & $\mathbf{0 . 1 0 1 9 8}$ & $\mathbf{0 . 1 6 1 0 4}$ & $\mathbf{0 . 1 1 4 7 9}$ & $\mathbf{0 . 0 8 3 5 9}$ & $\mathbf{0 . 0 9 5 6 2}$ \\
\hline
\end{tabular}

Table 5. Variation of cycle features

Also the last line of Table 5 is the different value of feet around the corresponding cycle time points. It can be seen that the variation of cycle feature of every group is less 0.20 , but the variety has no obvious trend as shown in Fig. 34.

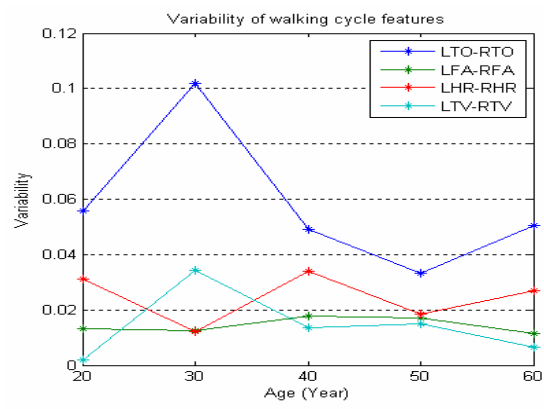

Fig. 34. Variation of cycle feature

\subsection{Orbital symmetry}

Orbital symmetry is described by the variability of the locus of symmetric points of body, in particular in the sagittal plane. Before doing calculation, we must do some adjustment about 
dynamic data of right side and left side. Adjustment method is to move the left side data ahead about half stride cycle. That is, we let the LIC to match the RIC. The first removal data may put to last part according to stride cycle, or be doffed and then we got three steps bilateral data.

It is clearly that selected features of symmetry may as indicators for evaluation of a person's gait. According to our work, the indices of motion data may be an immediate measure for quantification dynamic gait symmetry. The indices of up-body even the arm can be used as a factor to qualify the symmetry of human walking. The symmetry of gait should include footprint, cycle and dynamic data.

Normal walking does not need consider anything, but walking is very complex control, including central command, physical balance and coordination control, involving segments about feet, ankle, knee, hip, torso, neck, shoulder, arm and joint coordination. Any aspect of the disorder may affect gait symmetry, and some abnormalities may be compensatory or conceal. Pathological gait is often characterized by asymmetry, the selected frequencies of the movements of the limbs may deviate considerably from their eigenfrequencies and symmetry may be abandoned (Murray, 1967).

\subsection{Dynamic symmetry based on dynamic time warping}

In theory, the dynamic symmetry of gait is described by the properties which dynamic changed. These dynamic properties consist of three parts: position point symmetry, segment angle symmetry and joints angle symmetry.

\subsubsection{Definition of dynamic symmetry}

Theoretically, dynamic symmetry is described by the variability of the walking symmetric bilateral features. The dynamic symmetry is also composed of three parts, position symmetry $S(M o)$, segment symmetry $S(S A)$ and Joint symmetry $S(J A)$, as Equation (24).

$$
S(D)=S(M o)+S(S A)+S(J A)
$$

Here, $S(M o)=\sum_{i=1}^{8} \Delta\left(L P(t)_{i}, R P(t)_{i}\right), S(S A)=\sum_{i=1}^{5} \Delta\left(L \Phi(t)_{i}, R \Phi(t)_{i}\right), S(J A)=\sum_{i=1}^{5} \Delta\left(L \Theta(t)_{i}, R \Theta(t)_{i}\right)$.

And $L P(t) \in\{$ p1, p3, p5, p7, p9, p13, p15, p17 \}, RP(t) $\in\{$ p2, p4, p6, p8, p10, p14, p16, p18 \}, $\mathrm{L} \Phi(\mathrm{t}) \in\{\varphi 3, \varphi 5, \varphi 9, \varphi 11, \varphi 13\}, \operatorname{R} \Phi(\mathrm{t}) \in\{\varphi 4, \varphi 6, \varphi 10, \varphi 12, \varphi 14\}, \mathrm{L} \Theta(\mathrm{t}) \in\{\theta 3, \theta 5, \theta 7, \theta 9, \theta 11\}$, $R \Theta(t) \in\{\theta 4, \theta 6, \theta 8, \theta 10, \theta 12\}$.

The symbol $\Delta$ in the formulas will be described in next section. It is the DTW algorithm used to calculate the distance of two sequences with different length of them. Here consider all the symmetry properties included on upper body and lower body is want given the general definition of gait dynamic symmetry.

Considering 3-dimension, add $d=\{x, y, z\}$ to Equation (24), then we got Equation (25).

$$
S_{d}(D)=S_{d}(M o)+S_{d}(S A)+S_{d}(J A)
$$

Here Mo means motion data which has 8 attributes, $S A$ means Segment Angle data and JA means Joint Angle data, they both have 5 attributes. There is a total of 18 attributes.

Actually, we use the index like $S_{x}(M o), S_{y}(M o), S_{z}(M o)$ and $S_{x}(S A), S_{y}(S A), S_{z}(S A)$ and $S_{x}(J A)$, $S_{y}(J A), S_{z}(J A)$, not the $S_{d}(D)$.

The dynamic walking symmetry also has velocity and acceleration data. We got attributes as follow: $V M o \subset V(t), V S A \subset \Omega \Phi(t), V J A \subset \Omega \Theta(t), A M o \subset A(t), A S A \subset \Lambda \Phi(t), A J A \subset \Lambda \Theta(t)$. Then, we also can do calculation with three velocity equations. 
Similarly, it can easily be listed out three acceleration formulas. Thus, we have three sets of attributes, each containing 18 attributes. So, considering three directions, the dynamic symmetry may include $3 \times 18 \times 3=162$ indexes.

\subsubsection{Effects of aging on dynamic symmetry}

We use DTW algorithm to calculate the discrepancy between right data and left data on all attributes of all tested persons. The example of calculated result is shown as Fig. 35. We can see that somebody was more asymmetry than others on this attribute, for example number 2 and number 11 in $\mathrm{Z}$ direction.

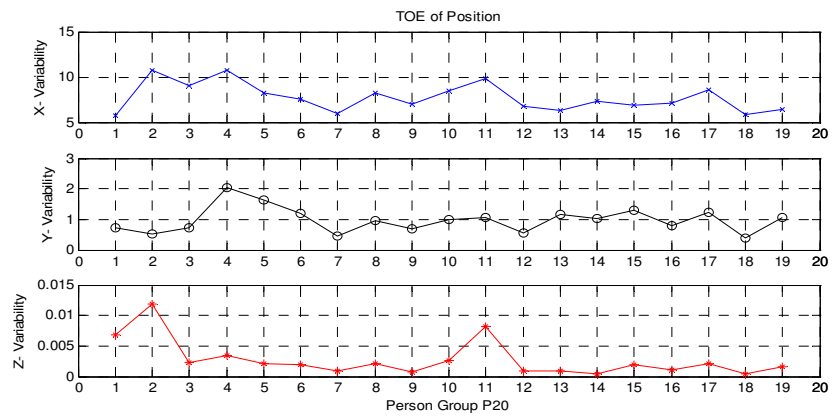

Fig. 35. Example of calculated result on one attribute

A total of 162 attribute needs for a similar calculation, to get the related discrepant values like Fig. 35. These results were used to do statistical analysis about gait symmetry.

\subsection{Feature selection on dynamic symmetry}

The dynamic symmetry is concerned with some direction features more meaningfully. Also some features did not reflect out gait characteristics goodly. Therefore, it is necessary in a large number of features to make a choice.

The existing gait symmetry indicators more used in accordance with the swing phase and stance phase, then neglected bilateral discrepancy of these phase while them were divided from cycles, thereby reducing the sensitivity of indicators.

We analyzed all results of the calculations and found that the dynamic symmetry is concerned with some direction indicators more meaningfully. Also some indicators did not reflect out gait characteristics goodly. Therefore, it is necessary in a large number of indicators to make a choice. In other words, it must do some feature selection.

Human walking is a complex procedure with both limb position variability and limb motion. When quantifying symmetry of walking gait, the used parameters and calculations should be chosen carefully (Karaharju-Huisnan, 2001)

\subsubsection{Selected symmetric features}

Although the tested persons are all healthy and walking with normal gait, but the individual may have a great difference in gait symmetry. So the general average method may result in larger bias, then it can think over use standard deviation.

Consider the three dimensional direction respectively, the gait symmetry data can available in three matrices. Then the rows of the matrix are the various attributes, and columns correspond 
to the tested persons of gait symmetry data. In this way we get three $54 \times n$ matrices and $n$ is person number. The original data come from the calculation results of bilateral discrepancy about symmetric attributes, and the calculation method was described in above sections.

For every attribute, that is the row of the matrix here, it can use the function $f a$ to calculate the minimal rate of discrepant value.

$$
f a=\frac{\bar{A}-A_{\min }}{A_{\max }-A_{\min }}
$$

Here $A$ also expresses the discrepant value on attribute $A$ of all persons. So $\bar{A}$ is the average value of all tested persons on attribute $A$, the $A_{\min }$ is the minimum value of the row, and the $A_{\text {max }}$ is the maximum value evidently.

It is clear, attribute $A$ is the element of the set which includes all symmetric attributes about 8 position items, 5 segment angle items, 5 joint angle items and their velocity and acceleration data.

The following table shows the comparable rates on $\mathrm{Z}$ direction, or namely sagittal direction.

\begin{tabular}{|c|c|c|c|c|c|c|c|c|}
\hline & SHO & ELB & WRI & HIP & KNE & ANK & HEE & TOE \\
\hline$M o$ & 0.166 & 0.157 & $\mathbf{0 . 0 3 0}$ & $\mathbf{0 . 0 3 3}$ & 0.113 & $\mathbf{0 . 0 7 3}$ & 0.151 & 0.180 \\
\hline$V M o$ & 0.408 & 0.177 & $\mathbf{0 . 0 5 9}$ & $\mathbf{0 . 0 2 9}$ & 0.453 & $\mathbf{0 . 0 6 3}$ & 0.289 & 0.292 \\
\hline$A M o$ & 0.266 & 0.305 & $\mathbf{0 . 0 4 2}$ & $\mathbf{0 . 0 2 4}$ & 0.262 & $\mathbf{0 . 0 4 6}$ & 0.292 & 0.176 \\
\hline
\end{tabular}

Table 6. Position attributes with velocity and acceleration

\begin{tabular}{|c|c|c|c|c|c|}
\hline & UPPERAR & FOREAR & THIGH & SHANK & FOOT \\
\hline$S A$ & 0.191 & $\mathbf{0 . 0 4 8}$ & $\mathbf{0 . 0 3 4}$ & $\mathbf{0 . 0 7 9}$ & 0.132 \\
\hline$V S A$ & 0.147 & 0.109 & $\mathbf{0 . 0 3 8}$ & $\mathbf{0 . 0 4 6}$ & 0.225 \\
\hline$A S A$ & 0.220 & $\mathbf{0 . 0 4 3}$ & $\mathbf{0 . 0 3 9}$ & $\mathbf{0 . 0 3 2}$ & 0.168 \\
\hline
\end{tabular}

Table 7. Segment angle attributes with velocity and acceleration

\begin{tabular}{|c|c|c|c|c|c|}
\hline & SHOULDER & ELBOW & HIP & KNEE & ANKLE \\
\hline$J A$ & 0.213 & 0.124 & $\mathbf{0 . 0 3 1}$ & 0.144 & 0.109 \\
\hline$V J A$ & 0.172 & 0.280 & 0.133 & $\mathbf{0 . 0 6 9}$ & 0.247 \\
\hline AJA & $\mathbf{0 . 0 4 6}$ & $\mathbf{0 . 0 4 2}$ & $\mathbf{0 . 0 3 7}$ & $\mathbf{0 . 0 4 8}$ & $\mathbf{0 . 0 3 1}$ \\
\hline
\end{tabular}

Table 8. Joint angle attributes with velocity and acceleration

There are 24 attributes in Table 6 to Table 8, and their rate less than 0.1 (see bold). In other words, these attributes have expressed better gait symmetry in this database. Also it can do same work on other two directions.

These attributes can be selected out for future testing a person whether or not symmetrical of his gait.

\subsubsection{Clustering on symmetry features for classification}

We use dynamic time warping (DTW) algorithm to calculate the similarity between bilateral symmetric attributes. Consider the three dimensional direction respectively, the gait 
symmetry data can available in three matrices. Then the rows of the matrix are the various attributes, and columns correspond to the tested persons of gait symmetry data. In this way we get three $54 \times n$ matrices (assumed we have $n$ valid test persons). The matrices data come from the calculation results of bilateral discrepancy about symmetric attributes.

Now we plan to do some clustering analysis, we need an algorithm which is no high cost on machine, but better on efficiency. Since there is a lot of clustering algorithms, for the sake of quickly carry out research and obtain some results, we have chosen the affinity propagation clustering algorithm APCLUSTER (Frey \& Dueck, 2007). The reason for using this algorithm is that it not only can do clustering on data but also can pass the original information rather than random values into the clustering processing, and meanwhile the algorithm has good performance and efficiency.

Using the APCLUSTER algorithm, we have done all the attributes clustering analysis in collusion with age or height or weight and so on. But in order to facilitate description, the following example is mainly discussed on age and gait symmetric data of sagittal direction. In addition, here we had 48 valid test objects, that is to say $n=48$.

Before the data entry the clustering algorithm function for computing, we also need to normalize the data, so that the two vectors may in the same range, and then the output graphics could be easier to see clearly with the clustering results. The normalizing ways and means are as follows.

$$
x_{i}{ }^{\prime}=\frac{x_{i}-\min A}{\max A-\min A}
$$

In the Equation (27), the $A$ is a vector and $x_{i}$ is its element. The $\min A$ is the minimum element of the vector and evidently the max $A$ is the maximum element of the vector. We get normalized element data as $x_{i}{ }^{\prime}$. That is, the original vector is $A=\left(x_{1}, x_{2}, \ldots, x_{n}\right)$, and then the normalized vector is $\mathrm{A}^{\prime}=\left(\mathrm{x}_{1}{ }^{\prime}, \mathrm{x}_{2}{ }^{\prime}, \ldots, \mathrm{x}_{\mathrm{n}}{ }^{\prime}\right)$.

Here are some examples of clustering of symmetric attributes. One clustering result is shown in Fig. 36. The inputs of the clustering algorithm are two vectors, one is the test person's gait kinetic data of Position Elbow and another is the corresponding age data.

In terms of our Fourteen-Linkage walking model, there are 54 symmetric attributes in one direction and they can be done clustering analysis. So there are 162 attributes in all for three directions. For each gait symmetric attribute to do clustering, the original symmetry data must be normalized to a vector. Take test person's age data to a vector, and take their gait data of one symmetric attribute to another vector. So the horizontal axis is normalized age data, and the vertical axis is normalized data of the symmetric attribute.

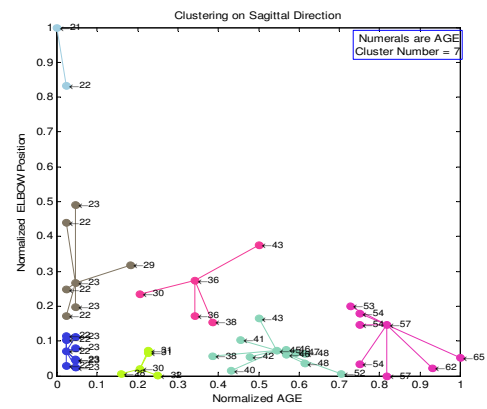

Fig. 36. Clustering on Position Elbow and Age 


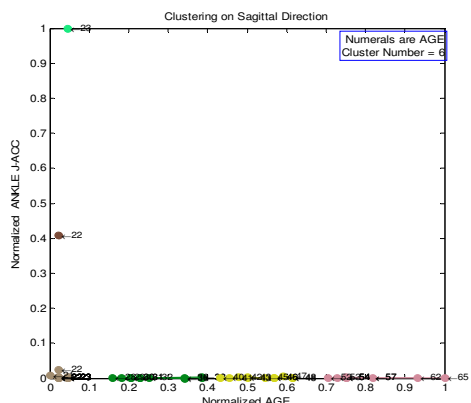

(a) Clustering on Acc of Joint Ankle and Age

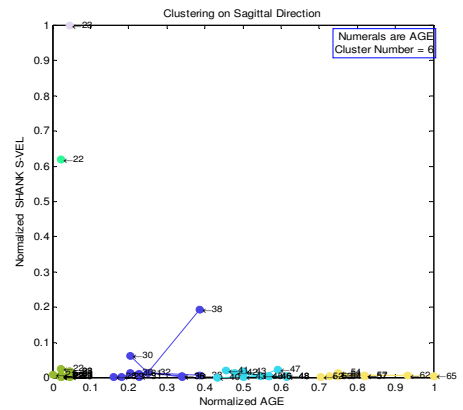

(b) Clustering on Vel of Segment Shank and Age

Fig. 37. More examples of clustering

For all the clustering results how to evaluate them. We used the following formula to calculate a mean square error. The formula is as follows.

$$
S A=\frac{1}{m} \sum_{i=1}^{m}\left(\frac{1}{n_{i}} \sum_{j=1}^{n_{i}} D\left(G_{i}(j)-C_{i}\right)\right)
$$

Here $m$ is the number of clustering centres or number of clustering groups, $n_{i}$ is the number of members of each group, and $C_{i}$ is the clustering centre of group $i$, while $G_{i}(j)$ denoted the member-point of the group $i$.

The distance calculation in the Equation (28) can use the Euclid's, such as the Equation (36).

$$
D_{1}\left(G_{i}(j)-C_{i}\right)=\sqrt[2]{\left(x_{G_{i}(j)}-x_{c_{i}}\right)^{2}+\left(y_{G_{i}(j)}-y_{c_{i}}\right)^{2}}
$$

Of course, the distance can be calculated using other methods, such as the absolute value of errors. This will be discussed later.

Use the Equation (28) with (29), we have calculated the values of all the clustering, choose the smallest ten values of the clustering attributes, and listed in Table 9.

\begin{tabular}{|l|l|l|l|}
\hline Attributes of Z-dir & No. & SA using $\mathrm{D}_{1}$ & Clustering \\
\hline THIGH S-ACC & 1 & 0.035657274 & 6 \\
\hline HIP J-ACC & 2 & 0.035669813 & 6 \\
\hline ANKLE J-ACC & 3 & 0.035717594 & 6 \\
\hline THIGH S-VEL & 4 & 0.035719691 & 6 \\
\hline HIP P-VEL & 5 & 0.035746144 & 6 \\
\hline SHANK S-ACC & 6 & 0.036075667 & 6 \\
\hline WRIST P-ACC & 7 & 0.036825407 & 6 \\
\hline SHANK S-VEL & 8 & 0.037846491 & 6 \\
\hline KNEE J-VEL & 9 & 0.03882457 & 6 \\
\hline WRIST Posit & 10 & 0.044141919 & 5 \\
\hline
\end{tabular}

Table 9. Top ten minimum values of clustering results 
From the Table 9, we can see that the smaller the SA value of 10 attributes has a relatively closer clustering results. In the Equation (28), if using other methods to calculate the distance, such as the Equation (30) and (31), we can compare their 10 minimum value of corresponds to the attributes and found that many of them are same, only individual attribute of exceptions, shown in Table 10. We followed the value of SA from small to large, listed from top to bottom.

$$
\begin{gathered}
D_{2}\left(G_{i}(j)-C_{i}\right)=a b s\left(y_{G_{i}(j)}-y_{c_{i}}\right) \\
D_{3}\left(G_{i}(j)-C_{i}\right)=\left(y_{G_{i}(j)}-y_{c_{i}}\right)^{2}
\end{gathered}
$$

Although every time the results of clustering is not exactly the same, but in the course of dozens of experiments, the attributes of the top ten were similar broadly, only slightly changed before and after the order. The values of these attributes are the 10 smallest of SA which value was calculated by three different distance formula $\mathrm{D}_{1}, \mathrm{D}_{2}$, and $\mathrm{D}_{3}$. So that you can more clearly see that most of these attributes are overlapped. This indicates that the relationship of that

\begin{tabular}{|c|c|c|c|c|c|}
\hline Attributes & SA using $D_{1}$ & Attributes & SA using $\mathrm{D}_{2}$ & Attributes & SA using $\mathrm{D}_{3}$ \\
\hline $\begin{array}{l}\text { THIGH S- } \\
\text { ACC }\end{array}$ & 0.035657274 & $\begin{array}{l}\text { THIGH S- } \\
\text { ACC }\end{array}$ & 0.00017294 & $\begin{array}{l}\text { THIGH S- } \\
\text { ACC }\end{array}$ & 0.000000074 \\
\hline HIP J-ACC & 0.035669813 & HIP J-ACC & 0.000523717 & $\begin{array}{l}\text { THIGH S- } \\
\text { VEL }\end{array}$ & 0.000001067 \\
\hline \begin{tabular}{|l} 
ANKLE J- \\
ACC \\
\end{tabular} & 0.035717594 & $\begin{array}{l}\text { THIGH S- } \\
\text { VEL }\end{array}$ & 0.000598035 & HIP J-ACC & 0.000003165 \\
\hline $\begin{array}{l}\text { THIGH S- } \\
\text { VEL }\end{array}$ & 0.035719691 & $\begin{array}{l}\text { ANKLE J- } \\
\text { ACC }\end{array}$ & 0.000894828 & $\begin{array}{l}\text { ANKLE J- } \\
\text { ACC }\end{array}$ & 0.000006696 \\
\hline HIP P-VEL & 0.035746144 & HIP P-VEL & 0.001258594 & HIP P-VEL & 0.000008664 \\
\hline \begin{tabular}{|l} 
SHANK S- \\
ACC \\
\end{tabular} & 0.036075667 & $\begin{array}{l}\text { SHANK S- } \\
\text { ACC }\end{array}$ & 0.001953265 & $\begin{array}{l}\text { SHANK S- } \\
\text { ACC }\end{array}$ & 0.000023426 \\
\hline \begin{tabular}{|l} 
WRIST P- \\
ACC \\
\end{tabular} & 0.036825407 & HIP P-ACC & 0.00276713 & WRIST Posit & 0.000090040 \\
\hline \begin{tabular}{|l} 
SHANK S- \\
VEL
\end{tabular} & 0.037846491 & $\begin{array}{l}\text { WRIST P- } \\
\text { ACC }\end{array}$ & 0.002879147 & \begin{tabular}{|l} 
ANKLE P- \\
VEL
\end{tabular} & 0.000131324 \\
\hline \begin{tabular}{|l|} 
KNEE \\
$\mathrm{J}-\mathrm{VEL}$
\end{tabular} & 0.03882457 & WRIST Posit & 0.005047818 & $\begin{array}{l}\text { WRIST P- } \\
\text { ACC }\end{array}$ & 0.000172132 \\
\hline WRIST Posit & 0.044141919 & \begin{tabular}{|l} 
SHANK S- \\
VEL
\end{tabular} & 0.00685193 & HIP Joint & 0.000225147 \\
\hline
\end{tabular}
using any distance formula and the selection of attributes was not so great.

Table 10. Comparison of three distance calculation

We can see Fig. 36 and Fig. 37 are different in shape and the latter are not so beautiful shape. These clusters are very closer to the center of all its members. Perhaps because of the presence of a large offset values, so most of the clusters are compressed in a small range. We can find that clustering results displaying isolated points of the cluster, actually these points show these persons with bad gait symmetry. It is maybe the method to classify asymmetry gait and symmetry gait. 


\section{Conclusions and discussions}

\subsection{Main conclusions}

In this paper, we have proposed a so-called FL model to analyze the walking stability and symmetry of different age subjects while walking on a normal pace. The most important finding is that human walking stabilities are not strictly monotone decreasing with age. Walking stability of human beings varies with age, but does not reduce in the elderly people always.

1. The variability of footprint increases with age for subjects over 30 years old, and it dramatically increases for the elderly over 60 years old, showing much less footprint stability.

2. The variability of walking cycle declines with age. That is to say, the elderly subjects have more cycle stability.

3. The variability of orbital increases with age for all subjects. In other words, the elderly has weaker orbital stability.

4. Human dynamic stability decreases with age except the twenties, which proves previous assumptions. This data mining technology not only gets the contributing dynamic stability features, but also makes the data acquisition simpler.

\subsection{Discussions in clinic}

Aging effects on motor control have been implicated as a key factor in adjusting posture during walking. Sensory feedback and muscular strength play important roles in maintaining stability against the presence of unpredictable external perturbations or internal variations of gait.

The footprint stability and walking stability of 20 years old subjects is less than that of over 30 years old. This cannot certify that 20 years old subjects have less quality of neuromuscular control. Nevertheless, they have much strength to control their walking pattern, so it shows a springily walking pattern. The orbital stability is strictly monotone decreasing with age. The orbital stability could express the ability of stability control more appropriately.

As we mentioned above, in most comprehensive opinions, walking stability will decrease with ageing. But the cycle stability increase with age. Why? It seems to be more confused to understand. In fact, in the three kinds of stability, only the cycle stability describes the relative stability of walking, which is the relative relationship among occurrence sequence of cycle events in a walking cycle, while the other two kinds of stability are absolute stability of posture. That indicates that the elderly subjects have a rigid and inflexible walking pattern. The elderly improves his/her walking stability by maintaining cycle stability more carefully, because it needs less strength to control cycle stability than the other two. That is to say, young subjects have more powerful muscles to control walking balance, while elderly subjects improve their walking stability by keeping their fixed walking patterns carefully. This is one of the most important findings of this paper.

One conclusion about gait symmetry is that, according to the attributes that selected out by APCLUSTER algorithm and our calculation analysis, we can classify some test objects in order to better meet the natural age groups. An appropriate grouping method to gait symmetry analysis will make the results of statistical analysis more meaningful.

Another conclusion is that, when the symmetry evaluation of normal walking gait, compared the trunk with the limbs, the latter gave larger contribution. Thus, if we have a device to measure gait symmetry of normal people, it may be wrong to wear it at the waist. It may very appropriate if we paste the device somewhere in the lower extremities (for example, shank or ankle), of course pair-wise and it will be more effective. 
The next study is to further deepen the existing clustering classification, including gait symmetry attributes and the relationship between weight and height in order to obtain meaningful results. It is our goal that combining gait symmetry attributes with a number of individual characteristics may construct a simple approach to determine a test object should belong to which group.

Clinical gait analysis is aimed at revealing a key aspect of abnormal gait and impact factors, so as to assist the rehabilitation assessment and treatment, but also help to assist the clinical diagnosis, evaluation. We hope that we can evaluate the symmetry degree of a person gait accurately, not whether symmetry or asymmetry. In other words, it can't use one piece of value, but use a set of indices to evaluate. And then each index may indicate an aspect of gait symmetry.

Further research needs to determine how these gait symmetry is related to actual fall risk. At least to a certain extent, the symmetry between the low-body such as legs seems to codetermine the stability of walking.

\section{Acknowledgment}

This work is supported in part by the Fukushima Prefectural Foundation for the Advancement of Science and Education (No.F-18-10), Japan, Shanghai, and Shanghai Leading Academic Discipline Project (J50103), China, and Pujiang Program from Science and Technology Commission of Shanghai Municipality, China.

The basic part of this work was implemented in the Biomedical Information Technology Lab, the University of Aizu, Fukushima, Japan.

\section{References}

Akita, K. (1984). Image Sequence Analysis of Real World Human Motion, Pattern Recognition, 17(1),1984, pp. 73-83

Arif, M. Ohtaki, Y. Nagatomi, R. \& Inooka, H. (2004). Estimation of the Effect of Cadence on Gait Stability in Young and Elderly People using Approximate Entropy Technique. Measurement Science Review, Volume 4, Section 2, 2004, pp.29-40

Cheng, J.C. \& Moura, J. M.F.(). Automatic Recognition of Human Walking in Monocular Image Sequences, Journal of VLSI Signal Processing Systems, 20(1-2), 1998, pp. 107-120

Chou, PH.; Chou, YL; Su, FC.; Huang, WK \& Lin, TS. (2003). Normal Gait of Children, Biomedical Engineeringapplications, Basis \& Communications, Vol. 15 No. 4 August 2003, pp. 160-163

Corriveau, H.; Hébert R.; Raîche, M. \& Prince, F. (2004). Evaluation of postural stability in the elderly with stroke, Archives of Physical Medicine and Rehabilitation, Vol 85, Issue 7, pp.1095-1101

Cunado, D.; Nixon, M.S. \& Carter, J.N. (2003). Automatic Extraction and Description of Human Gait Models for Recognition Purposes, Computer Vision and Image Understanding, Vol.90, No.1, (April 2003), pp. 1-41, ISSN 1077-3142

Dockstader, S. L.; Bergkessel, K. A. \& Tekalp A. M., Feature Extraction for the Analysis of Gait and Human Motion, Proc. of 16th International Conference on Pattern Recognition, Canada, 2002,pp.5-8.

Frey, B.J. \& Dueck, D. (2007). Clustering by passing messages between data points, Science, Vol 315, No 5814, pp 972-976, February 2007

George, F. (2000). Falls in the Elderly, American Family Physician, Apr. 2000

Guo, Y.; Xu, G. \& Tsuji, S. (1994). Understanding Human Motion Patterns, Proc. The 12th IAPR International Conference on Pattern Recognition. Vol.2, 1994, pp. 325-329 
Hylton B.M.; Stephen, R. L. and Richard, C. F. (2003). Age-related differences in walking stability. Age and Ageing 2003, 32, pp.137-142

Karaharju-Huisnan, T.; Taylor, S.; Begg, R.; Cai, J. \& Best, R. (2001). Gait symmetry quantification during treadmill walking, The Seventh Australian and New Zealand Intelligent Information Systems Conference, 18-21, Nov. 2001, pp. 203 - 206

Karaulova, I.A.; Hall, P.M. \& Marshall, A.D.(2000). A Hierarchical Model of Dynamics for Tracking People with a Single Video Camera, Proc of the 11th BMVC, 2000, pp. 262-352

Kavanagh, J. (2006). Dynamic stability of the upper body during walking. PhD thesis, School of Physiotherapy and Exercise Science, Griffith Health, Griffith University, 2006.

Lee, L. (2003). Gait Analysis for Classification, AI Technical Report 2003-014, Massachusetts Institute of Technology-artificial Intelligence Laboratory, 2003.

Li, Y.; Wen, C.L.; Xie, Z. \& Xu, X.H. (2003). Synchronization of batch trajectory based on multi-scale dynamic time warping, Proceeding of the Second International Conference on Machine Learning and Cybernetics, 2003.

Marin, L.C.; Kang, H.G. \& Dingwell, J.B. (2006). Changes in the Orbital Stability of Walking Across Speeds, Proceedings of the 30th Annual Meeting of the American Society of Biomechanics, Blacksburg, VA, September 6-9, 2006.

Menz HB. (2002). Walking Stability in Young, Old and Neuropathic Subjects, PhD thesis, School of Physiology and Pharmacology, Faculty of Medicine, University of New South Wales, 2002.

Murray, M.P. (1967). Gait as A Total Pattern of Movement, American Journal of Physical Medicine, 46(1), 1967, pp. 290-332.

Nash, J.; Carter, J.N. \& Nixon, M.S. (1998). Extraction of Moving Articulated Objects by Evidence Gathering, Proc. of the 9th British Machine Vision Conference, ISBN 9781901725049, Southampton, UK, September 14-17, 1998, pp. 609-618

Nichols, D.S. Balance retraining after stroke using force platform biofeedback. Phys Ther. 1997 May; 77(5), pp. 553-8

Rohr, K. (1994). Towards model-based recognition of human movements in image sequences", CVGIP: IU, 74(1), 1994, pp. 94-115

Stirling, J.R. \& Zakynthinaki, M.S. (2004). Stability and the maintenance of balance following a perturbation from quiet stance, Chaos: An Interdisciplinary Journal of Nonlinear Science, March 2004, Volume 14, Issue 1, pp. 96-105

Sutherland, D.H.; Olshen, R.A.; Cooper, L. \& Woo, SLY. (1980). The Development of Mature Walking, Bone Joint Surg, 1980,Vol. 62A, No. 3, pp 336-353

Whittle, M. W. (2007). Gait Analysis: An Introduction, (4th ed), Elsevier Health Sciences, ISBN 9780750688833, New York

Woollacott, M.H. \& Tang, P.F. (1997). Dynamic Balance Control During Walking in the Older Adult: Research and its Implications, Physical Therapy, 7(6), 1997, pp. 646-660

Yang N.F. (2001). Coordination Analysis and Parametric Description of Human Movements, doctoral thesis, Tsinghua University, 2001

Yoo, J. H.; Nixon, M. S \& Harris, C. J. (2002). Extracting Gait Signatures based on Anatomical Knowledge. Proc. of BMVA Symposium on Advancing Biometric Technologies, 2002.

Zhang, R.; Vogler, C. \& Metaxas, D. (2004). Human Gait Recognition, Proceedings of the 2004 IEEE Computer Society Conference on Computer Vision and Pattern Recognition Workshops (CVPRW'04), 2004, pp.1-8 


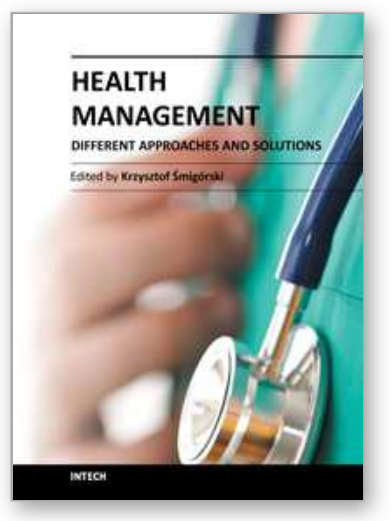

\author{
Health Management - Different Approaches and Solutions \\ Edited by Dr. Krzysztof Smigorski
}

ISBN 978-953-307-296-8

Hard cover, 498 pages

Publisher InTech

Published online 14, December, 2011

Published in print edition December, 2011

The development in our understanding of health management ensures unprecedented possibilities in terms of explaining the causes of diseases and effective treatment. However, increased capabilities create new issues. Both, researchers and clinicians, as well as managers of healthcare units face new challenges: increasing validity and reliability of clinical trials, effectively distributing medical products, managing hospitals and clinics flexibly, and managing treatment processes efficiently. The aim of this book is to present issues relating to health management in a way that would be satisfying for academicians and practitioners. It is designed to be a forum for the experts in the thematic area to exchange viewpoints, and to present health management's stateof-art as a scientific and professional domain.

\title{
How to reference
}

In order to correctly reference this scholarly work, feel free to copy and paste the following:

Bofeng Zhang, Susu Jiang, Ke Yan and Daming Wei (2011). Human Walking Analysis, Evaluation and Classification Based on Motion Capture System, Health Management - Different Approaches and Solutions, Dr. Krzysztof Smigorski (Ed.), ISBN: 978-953-307-296-8, InTech, Available from: http://www.intechopen.com/books/health-management-different-approaches-and-solutions/human-walkinganalysis-evaluation-and-classification-based-on-motion-capture-system

\section{INTECH}

open science | open minds

\author{
InTech Europe \\ University Campus STeP Ri \\ Slavka Krautzeka 83/A \\ 51000 Rijeka, Croatia \\ Phone: +385 (51) 770447 \\ Fax: +385 (51) 686166 \\ www.intechopen.com
}

\author{
InTech China \\ Unit 405, Office Block, Hotel Equatorial Shanghai \\ No.65, Yan An Road (West), Shanghai, 200040, China \\ 中国上海市延安西路65号上海国际贵都大饭店办公楼 405 单元 \\ Phone: +86-21-62489820 \\ Fax: +86-21-62489821
}


(C) 2011 The Author(s). Licensee IntechOpen. This is an open access article distributed under the terms of the Creative Commons Attribution 3.0 License, which permits unrestricted use, distribution, and reproduction in any medium, provided the original work is properly cited. 